\title{
On controllability of second order dynamical systems - a survey
}

\author{
J. KLAMKA*, J. WYRWAE, and R. ZAWISKI \\ Silesian University of Technology, 16 Akademicka St., 44-100 Gliwice, Poland
}

\begin{abstract}
The paper presents a survey of recent results in the area of controllability of second order dynamical systems. Controllability problem for finite and infinite dimensional, linear, semilinear, deterministic and stochastic dynamical systems (with delays and undelayed) is taken into consideration. Different types of controllability are discussed.
\end{abstract}

Key words: exact controllability, approximate controllability, second order dynamical systems, infinite dimensional systems, semilinear systems.

\section{Introduction}

Controllability is one of the most fundamental concepts in mathematical control theory. It is a qualitative property of dynamical systems important from theoretical as well as practical point of view. The notion of controllability, introduced in the early sixties of the 20th century by Kalman, covers time-invariant and time-varying linear control systems described by a state equation [1-5].

Roughly speaking, controllability means that it is possible to steer, in a given time interval, a dynamical system from any arbitrary initial state to any arbitrary final state by means of admissible controls. In general, there are many different definitions of controllability, the applications of which depend on the class of dynamical system.

Controllability has many practical implications in control theory. There are strong relationships between controllability and such concepts as stability and stabilizability, observability and detectability of linear both finite-dimensional and infinite-dimensional control systems. The problem of observability is related to the fact that in real world systems, not all state variables are available for direct measurements. In practice, the number of state variables is much greater than the number of outputs. In this context, observability denotes studying the possibility of estimating the state of the dynamical system from the output. If the dynamical system is observable, all modes of the system may be observed from the output. The concept of observability is strongly related to the state observers of dynamical systems. Controllability and observability are dual concepts; a dynamical system is controllable if and only if the corresponding dual system is observable and vice versa $[6,7]$. The duality between controllability and observability makes it possible to reduce optimal control problems to optimal observation problems and vice versa [4]. Detectablility means the observability of at least all unstable modes of the dynamical system. If all modes of the

*e-mail: jerzy.klamka@polsl.pl dynamical system are unstable, the concepts of observability and detectability are equivalent. On the other hand, stabilizability means controllability of at least all unstable modes of the dynamical system. Once again, if all modes of the system are unstable, notions of controllability and stabilizability coincide. If the system is stabilizable, it is possible to stabilize it by the appropriate state feedback, whereas if the system is stabilizable and detectable, it is possible to stabilize it by the appropriate output feedback. In the same way as for controllability and observability, there is a formal duality between stabilizability and detectability concepts.

Controllability is also strongly related to the theory of realization and so called minimal realization [8-10]. Realization theory makes clear the relationship between input-output description in the operator domain (transfer-function) and statespace representation (system of differential equations) in the time domain. The aim of the minimal state-space realization problem is to find a state-space model of minimal size of the given system. Realization theory has also shown the importance of using the right system model for a particular problem. The transfer function formulation does not reveal the behavior inside the system, such as unobservable unstable modes. Therefore, the transfer function matrix cannot always be used to study the stability properties of the time-invariant systems, since it describes only the controllable and observable part of the dynamical system. This is because of so-called hidden polezero cancellations $[11,4]$. Different properties of dynamical systems stand out more clearly in some special descriptions of the system referred to as canonical forms. That is why they are often used to simplify the system analysis and synthesis of the solution to many problems of control theory. Controllability is strongly related to Kalman, Jordan and Luenberger canonical forms for linear time-invariant control systems [3, 12, 13]. Moreover, the concept of controllability plays an important role in determining the solution to the optimal control problem, in particular to the so called minimum energy control problem for many classes of linear finite dimensional, infinite dimensional and delayed dynamical systems, both deterministic and stochastic [14-17]. Therefore, controllability criteria are useful 
in determining the solution to the following problems of control theory:

- stabilizability conditions,

- duality theorems, concerning in particular observability and detectability conditions,

- solution to the optimal control problems, in particular to the minimum energy control problem,

- transforming the system to equivalent canonical forms (e.g. Kalman, Jordan, Luenberger forms).

In this paper, an overview of the current state of controllability analysis for the second order linear and semilinear infinite dimensional dynamical systems is given. The paper is focused on various types of controllability which suit the types of systems they describe. In doing so, it is possible to give an overview of various approaches and techniques used in this problem. Among the considered controllability types and, what follows, types of systems analysed, there are approximate controllability of second order linear infinite dimensional systems, approximate controllability of second order semilinear finite dimensional systems with delay in control, trajectory controllability of second order semilinear integro-differential systems, approximate controllability of a second order neutral stochastic differential equations with state dependent delay, nonlocal conditions and Poisson jumps, exact controllability of Duffing equation, exact boundary controllability of coupled hyperbolic equations and exact controllability of neutral integrodifferential impulsive systems with infinite delay and damping.

The paper is organized as follows. In the Preliminaries section we give the basic definitions, together with necessary notation, to form a common basis to which other section refer. The Controllability Results section forms the main part of this survey. Each of the system types is described there together with appropriate comments on its controllability results. The concluding section gives a notion about the direction of research in the field of controllability of second order dynamical systems.

\section{Preliminaries}

As stated in the introduction, this section contains common notions used throughout the article. To maintain the appropriate level of generality, the definitions below are given with reference to Banach spaces.

Consider a dynamical system described by the general state equation defined on the closed time interval $J=[0, T]$ as:

$$
\frac{d}{d t} x(t)=\mathfrak{f}(t, x(t), u(t)),
$$

that is, the equation describing the dependance between the time change of a state of system $x(t) \in X$ under the influence of the admissible control signal $u(t) \in U$ and possibly other arguments where $X$ and $U$ are Banach spaces. The generally nonlinear function $\mathfrak{f}$ on the right-hand side of (1) differs in nature and the list of arguments in each of the cases under discussion, thus defining the character of the system. It is generally assumed that function $f$ is such that equation (1) has a solution (not necessary unique) for suitably defined initial condition $x(0) \in X$.

The solution to (1) mostly takes the form of an integral equation, referred to as a mild solution [18]:

$$
x(t)=\int_{0}^{t} \mathfrak{F}(t, u(t)) d s, t \in[0, T],
$$

which is a starting point to the analysis of controllability [19].

The term reachable set (or attainable set) is closely connected with (2) and is given by the following definitions.

Definition 2.1. Reachable set. The reachable set $R_{T}(x(0)) \subseteq X$ is the set of all values which are reachable from initial state $x(0)$ by the system state $x(t)$ with the use of admissible controls $u(t)$ in a given time interval $[0, T]$. For $x(0)=0$ the set $R_{T}(x(0))$ is denoted shortly as $R_{T}$.

With the notion of reachable set one can formulate the following

Definition 2.2. Approximate controllability. The system described by (2) is called approximately controllable on $[0, T]$ when the reachable set $R_{T}(x(0))$ is dense in $X$.

Definition 2.3. Exact controllability. The system described by (2) is called exactly controllable on $[0, T]$ when the reachable set $R_{T}(x(0))$ in equal to $X$.

Apart from the above definitions, the following are also common for some of the system types described below.

\section{Definition 2.4. Space of piecewise continuous functions.}

(i) A function $x:[\sigma, \tau] \rightarrow X$ is said to be a normalized piecewise continuous function on $[\sigma, \tau]$ if $x$ is a piecewise continuous and left continuous on $[\sigma, \tau]$.

(ii) The space $P C([\sigma, \tau], X)$ is referred to as the space of normalized piecewise continuous functions from $[\sigma, \tau]$ into $X$. In particular, the space $P C$ is formed by all normalized piecewise continuous functions $x: J \rightarrow X$ such that $x(\cdot)$ is continuous at $t \neq t_{k}, x\left(t^{-}\right)=x\left(t_{k}\right)$ and $x\left(t_{k}^{+}\right)$exists for $k=1,2,3, \ldots, m$.

The abstract phase space $\mathfrak{B}$ is defined axiomatically $[20,21]$.

Definition 2.5. Phase space $\mathfrak{B}[22]$. The phase space $\mathfrak{B}$ is the vector space of functions defined from $(-\infty, 0]$ into $X$ endowed with a seminorm denoted $\|\cdot\|_{\mathfrak{B}}$ and such that the following axioms hold:

(A) If $x:(-\infty, \mu+b] \rightarrow X, b>0$ is such that $x_{\mu} \in \mathfrak{B}$ and $\left.x\right|_{[\mu, \mu+b]} \in P C([\mu, \mu+b], X)$, then for every $t \in[\mu, \mu+b)$, the following hold:

(i) $x_{t}$ is in $\mathfrak{B}$,

(ii) $\|x(t)\|_{\mathfrak{B}} \leq H\left\|x_{t}\right\|_{\mathfrak{B}}$,

(iii) $\left\|x_{t}\right\|_{\mathfrak{B}} \leq K(t-\mu) \sup \{\|x(s)\|: \mu \leq s \leq t\}+M(t-\mu)$ $\left\|x_{\mu}\right\|_{\mathfrak{B}}$, where $H>0$ is a constant; $K, M:[0, \infty)$ $\rightarrow[1, \infty), K$ is continuous, $M$ is locally bounded and $H, K, M$ are independent of $x(\cdot)$. 
(B) The space $\mathfrak{B}$ is complete.

Consider also the following definition of an evolution system.

Definition 2.6. Evolution system [22]. A family $\mathfrak{S}$ of bounded linear operators $\mathfrak{S}(t, s): J \times J \rightarrow \mathscr{L}(X)^{1}$ is called an evolution operator with an infinitesimal closed densely defined generator $A(t): \mathscr{D}(A(t)) \subseteq X \rightarrow X, t \in J$, if the following conditions are satisfied:

(Z1) For each $x \in X$ the mapping $J \times J \ni(t, s) \rightarrow \mathfrak{S}(t, s) x \in X$ is of $C^{1}$ class and:

(i) for each $t \in J, \mathfrak{S}(t, t)=0$,

(ii) for all $t, s \in J$ and for each $x \in X$,

$$
\left.\frac{\partial}{\partial t} \mathfrak{S}(t, s) x\right|_{t=s}=x,\left.\quad \frac{\partial}{\partial s} \mathfrak{S}(t, s) x\right|_{t=s}=-x \text {. }
$$

(Z2) For all $t, s \in J$, if $x \in \mathscr{D}(A)$, then $\mathfrak{S}(t, s) x \in \mathscr{D}(A)$, the mapping $J \times J \ni(t, s) \rightarrow \mathfrak{S}(t, s) x \in X$ is of $C^{2}$ class and:
(i) $\frac{\partial^{2}}{\partial t^{2}} \mathfrak{S}(t, s) x=A(t) \mathfrak{S}(t, s) x$,
(ii) $\frac{\partial^{2}}{\partial t^{2}} \mathfrak{S}(t, s) x=\mathfrak{S}(t, s) A(s) x$,
(iii) $\left.\frac{\partial}{\partial s} \frac{\partial}{\partial t} \mathfrak{S}(t, s) x\right|_{t=s}=0$.

(Z3) For all $t, s \in J$, if $x \in \mathscr{D}(A)$, then $\frac{\partial}{\partial s} \mathfrak{S}(t, s) x \in \mathscr{D}(A)$, there exist $\frac{\partial^{2}}{\partial t^{2}} \mathfrak{S}(t, s) x, \frac{\partial^{2}}{\partial s^{2}} \frac{\partial}{\partial t} \mathfrak{S}(t, s) x$ and:

(i) $\frac{\partial^{2}}{\partial t^{2}} \frac{\partial}{\partial s} \mathfrak{S}(t, s) x=A(t) \frac{\partial}{\partial s} \mathfrak{S}(t, s) x$,

(ii) $\frac{\partial^{2}}{\partial s^{2}} \frac{\partial}{\partial t} \mathfrak{S}(t, s) x=\frac{\partial}{\partial t} \mathfrak{S}(t, s) A(s) x$,

and the mapping $J \times J \ni(t, s) \rightarrow A(t) \frac{\partial}{\partial s} \mathfrak{S}(t, s) x$ is continuous.

\section{Controllability results for second-order linear infinite-dimensional dynamical systems}

Up to the present time, the problem of controllability of continuous and discrete time linear dynamical systems has been extensively investigated in many papers [7, 23-29]. Different types of linear systems have been investigated including time-invariant and time-varying systems, finite-dimensional and infinite dimensional systems as well as systems with unconstrained and constrained controls. In the case of most of semilinear dynamical systems, controllability criteria are formulated in such a way that an overall system may be controllable only if the linear part of semilinear system is controllable. To verify controllability for a class of linear second order systems, the criteria presented in this section may be used.

Consider linear infinite-dimensional control system described by the following abstract second-order differential equation:

${ }^{1} \mathscr{L}(X, Y)$ is the space of all bounded operators from $X$ into $Y$, simply $\mathscr{L}(X)$ if $X=Y$

$$
\begin{aligned}
& \left(e_{2} A+e_{1} A^{\frac{1}{2}}+e_{0} I\right) \frac{d^{2}}{d t^{2}} v(t)+ \\
& +2\left(c_{2} A+c_{1} A^{\frac{1}{2}}+c_{0} I\right) \frac{d}{d t} v(t)+ \\
& +\left(d_{2} A+d_{1} A^{\frac{1}{2}}+d_{0} I\right) v(t)=B u(t) \\
& v(0) \in \mathscr{D}(A), \frac{d}{d t} v(0) \in V
\end{aligned}
$$

where:

1. $V$ and $U$ denote separable Hilbert spaces,

2. $e_{2} \geq 0, e_{1} \geq 0, e_{0} \geq 0, e_{2}+e_{1}+e_{0}>0, c_{2} \geq 0, c_{1} \geq 0$, $c_{0} \geq 0, d_{1}$ and $d_{0}$ unrestricted in sign, $d_{2}>0$ are given constants,

3. $A: V \supset \mathscr{D}(A) \rightarrow V$ is a linear generally unbounded self-adjoint and positive-definite linear operator with domain $\mathscr{D}(A)$ dense in $V$ and compact resolvent,

4. operator $B$ is linear and bounded from the space $U$ into the space $V$,

5. controls $u$ belong to the set of admissible controls $L_{l o c}^{2}([0, \infty), U)$,

Introduce the product space $W=\mathscr{D}\left(A^{\frac{1}{2}}\right) \times V$ with the inner product:

$\langle v, w\rangle_{W}=\left\langle\left[v_{1}, v_{2}\right],\left[w_{1}, w_{2}\right]\right\rangle_{W}=\left\langle A^{\frac{1}{2}} v_{1}, A^{\frac{1}{2}} w_{1}\right\rangle_{V}+\left\langle v_{2}, w_{2}\right\rangle_{V}$.

Using standard substitution:

$$
v(t)=\left[\begin{array}{l}
w_{1}(t) \\
w_{2}(t)
\end{array}\right]=\left[\begin{array}{l}
v(t) \\
\dot{v}(t)
\end{array}\right]
$$

system (3) may be rewritten as the equivalent first order dynamical system in the product space $W$ described by the following first order differential equation:

$$
\frac{d}{d t} w(t)=F w(t)+G u(t)
$$

where:

$$
\begin{aligned}
& F=\left[\begin{array}{cc}
0 & I \\
F_{21} & F_{22}
\end{array}\right], \\
& F_{21}=-\left(e_{2} A+e_{1} A^{\frac{1}{2}}+e_{0} I\right)^{-1}\left(d_{2} A+d_{1} A^{\frac{1}{2}}+d_{0} I\right) \\
& F_{22}=-2\left(e_{2} A+e_{1} A^{\frac{1}{2}}+e_{0} I\right)^{-1}\left(c_{2} A+c_{1} A^{\frac{1}{2}}+c_{0} I\right) \\
& G=\left[\begin{array}{c}
0 \\
\left(e_{2} A+e_{1} A^{\frac{1}{2}}+e_{0} I\right)^{-1} B
\end{array}\right] .
\end{aligned}
$$

Remark 1. From assumptions on operator $A$ it follows that the operator $\left(e_{2} A+e_{1} A^{\frac{1}{2}}+e_{0} I\right)^{-1}$ is sef-adjoint, positive and bounded on $\mathrm{V}$. 
In addition to the second-order equation (3) consider a simplified first-order differential equation:

$$
\frac{d}{d t} v(t)=A^{\alpha} v(t)+B u(t)
$$

where $\alpha \in(0, \infty)$.

Definition 3.1. (Approximate controllability). Dynamical system (5) is said to be approximately controllable at the time interval $[0, T]$ if for any initial condition $w(0) \in W$, any given final condition $w_{f} \in W$ and each positive real number $\varepsilon$, there exists an admissible control $u \in L_{l o c}^{2}((0, T), U)$ such that:

$$
\left\|w(T ; w(0), u)-w_{f}\right\|_{W} \leq \varepsilon .
$$

Definition 3.2. (Approximate controllability at a finite time). Dynamical system (5) is said to be approximately controllable at a finite time if for any initial condition $w(0) \in W$, any given final condition $w_{f} \in W$ and each positive real number $\varepsilon$, there exists a finite time $T<\infty$ (depending generally on $w(0)$ and $w_{f}$ and an admissible control $u \in L_{\text {loc }}^{2}((0, T], U)$ such that inequality (8) holds.

Lemma 3.1. Approximate controllability [30]. Dynamical system (7) is approximately controllable in a finite time for each $\alpha \in(0, \infty)$ if and only if it is approximately controllable in a finite time for some $\alpha \in(0, \infty)$.

Lemma 3.2. Approximate controllability [31]. Dynamical system (5) is approximately controllable in a finite time if and only if for any complex number $z$, there exists no nonzero $w \in D\left(F^{*}\right)$ such that:

$$
\left[\begin{array}{c}
F^{*}-z I \\
G^{*}
\end{array}\right]=0 .
$$

Similarly, dynamical system (7) is approximately controllable in a finite time if and only if for any complex number $s$, there exists no nonzero $v \in D\left(A^{\alpha}\right) \subset V$ such that:

$$
\left[\begin{array}{c}
-A^{\alpha}-s I \\
B^{*}
\end{array}\right]=0 .
$$

Operators $A^{*}, G^{*}$ and $B^{*}$ are adjoint operators for $F, G$ and $B$, respectively.

Theorem 3.3. Approximate controllability [32]. Dynamical system (3) is approximately controllable in a finite time if and only if dynamical system (7) is approximately controllable in a finite time for some $\alpha \in(0, \infty)$.

Proof of Theorem 3.3 is based on a perturbation theory of controllable systems.

Remark 2. Results of Theorem 3.3 make it possible to verify approximate controllability of second-order linear dynamical systems on the basis of a well-known approximate controllability criteria for first-order dynamical systems.

\section{Controllability results for semilinear dynamical systems}

The controllability concept for nonlinear systems are intensively studied in literature [33-39]. The semilinear dynamical systems are special case of nonlinear dynamical systems. They consist of purely linear and purely nonlinear parts in differential state equations. One of the most popular approach to controllability analysis for these systems is to treat a nonlinear part of the semilinear system as a nonlinear perturbation of linear system. This research direction is particularly attractive since it allows to determine if the semilinear system is controllable by verifying controllability for much simpler linear part of semilinear system and checking some additional, rather simply verifiable, conditions assuring boundedness of nonlinear part of the system. Another popular method of studying controllability of these systems is based on different fixed point theorems.

4.1. Constrained controllability of second order semilinear systems with delay. Modelling and control of dynamical systems with delays arise naturally in numerous engineering problems. Satisfactory modelling of time delays appearing in a real systems is also important for the synthesis of effective control systems since systems with delays show significantly different characteristics from those without delays. As a consequence, the presence of time delay is often the cause of substantial performance deterioration and even instability of the system. That is why controllability of time-delay dynamical systems has been studied in a great number of papers recently [40, 41, 38, 42, 39]. It is also motivated by significant theoretical problems posed by such systems. It has to be emphasized that in a time-delay system, systems with point and distributed delays, systems with delays in a state variable and in a control can be distinguished. Moreover, in semi-linear dynamical systems delays may be contained both in purely linear and purely nonlinear parts of differential state equation.

Sufficient conditions for constrained local relative controllability near the origin in a prescribed finite time interval for semi-linear dynamical systems with multiple variable point delays or distributed delays in a control and in state variables with a nonlinear term continuously differentiable near the origin are presented in [42] and [39].

Consider semilinear finite-dimentional control system with single point delay described by a following differential equation:

$$
\begin{aligned}
\frac{d^{2}}{d t^{2}} w(t) & =G w(t)+f(w(t), u(t), u(t-h))+ \\
& +H u(t)+K u(t-h) \text { for } t \in[0, T], \\
w(0)=0, & \frac{d}{d t} w(0)=0, u(t)=0 \text { for } t \in[-h, 0],
\end{aligned}
$$

where state vector $w(t) \in R^{n}=W$ and control vector $u(t) \in U$, $G$ is $n \times n$ dimensional constant matrix, $H$ and $K$ are $n \times m$ dimensional constant matrices, $H>0$ is single point delay.

Moreover, it is assumed that a nonliner mapping $f: W \times$ $U \times U \rightarrow W$ is continuously differentiable near the origin and such that $f(0,0,0)=0$. 
Using standard substitution:

$$
x(t)=\left[\begin{array}{l}
x_{1}(t) \\
x_{2}(t)
\end{array}\right]=\left[\begin{array}{l}
w(t) \\
\dot{w}(t)
\end{array}\right]
$$

system (11) may be rewritten as an equivalent first order semilinear stationary $2 n$-dimensional equation described by a following semilinear ordinary differential equation:

$$
\begin{aligned}
\frac{d}{d t} x(t) & =A x(t)+F(x(t), u(t), u(t-h))+ \\
& +B u(t)+D u(t-h)
\end{aligned}
$$

for $t \in[0, T]$ with zero initial condition:

$$
x(0)=0, u(t)=0 \text { for } t \in[-h, 0],
$$

where $x(t) \in R^{2 n}=X$ and a control $u(t) \in R^{m}=U, A$ is $2 n \times 2 n$ dimensional constant matrix, $F \in R^{2 n}, B$ and $D$ are $2 n \times m$ dimensional constant matrices of the following form:

$$
\begin{aligned}
& A=\left[\begin{array}{ll}
0 & I \\
G & 0
\end{array}\right], B=\left[\begin{array}{l}
0 \\
H
\end{array}\right], D=\left[\begin{array}{l}
0 \\
K
\end{array}\right], \\
& F(x(t), u(t), u(t-h))=\left[\begin{array}{c}
0 \\
f(w(t), u(t), u(t-h))
\end{array}\right] .
\end{aligned}
$$

Remark 3. From assumptions on $f(w(t), u(t), u(t-h))$ it follows that a nonlinear mapping $F: X \times U \times U \rightarrow X$ is also continuously differentiable near the origin and such that $F(0,0,0)=0$.

The set of admissible controls for control systems (11) and (13) is defined as $U_{a d}=L_{\infty}\left([0, T], U_{c}\right)$ where $U_{c} \subset U$ is a given closed and convex cone with nonempty interior and vertex at zero.

For a given admissible control $u(t) \subset U_{a d}$ there are unique solutions $w(t, u) \in R^{n}$ and $x(t, u) \in R^{2 n}$ to the second order and first order differential equations (11) and (13) with zero initial condition given by the following integral formula [40]:

$$
\begin{aligned}
x(t, u) & =\int_{0}^{t} S(t-s)(F(x(s, u(s)), u(s), u(s-h))+ \\
& +B u(s)+D u(s-h)) d s
\end{aligned}
$$

where matrix $S(t)=\exp (A t)$ for $t \geq 0$ is $2 n \times 2 n$ dimensional exponential transition matrix for the linear part of the semilinear first order control system (13).

Definition 4.1. Reachable set [40]. The attainable set $R_{T}\left(U_{c}\right)$ for the dynamical system (13) at the given final time $T>0$ from zero initial conditions is defined as follows:

$$
\begin{array}{r}
R_{T}\left(U_{c}\right)=\left\{x \in X: x=x(T, u), u(t) \in U_{c}\right. \\
\text { for a.e. } t \in[0, T]\}
\end{array}
$$

where $x(t, u), t>0$ is a unique solution to a first order differential equation (13) given by (16) with zero initial conditions and given set of admissible controls $u \in U_{a d}=L_{\infty}\left([0, T], U_{c}\right)$.

In literature the notion of reachable set is often referred to as an attainable set.

The two basic types of controllability are given by the following definitions.

Definition 4.2. $\boldsymbol{U}_{c}$-local controllability [40]. The control system (11) is said to be $U_{c}$-locally controllable in $[0, \mathrm{~T}]$ if the attainable set $R_{T}\left(U_{c}\right)$ contains a neigborhood of zero in the space $X$.

Definition 4.3. $\boldsymbol{U}_{\boldsymbol{c}}$-global controllability [40]. The control system (11) is said to be $U_{c}$-globally controllable in $[0, \mathrm{~T}]$ if $R_{T}\left(U_{c}\right)=X$.

Let a linear $2 n$ dimensional control system given by

$$
\frac{d}{d t} z(t)=C z(t)+E u(t)+G u(t-h) \text { for } t \in[0, T]
$$

with zero initial condition $z(0)=0$ and $u(t)=0$ for $t \in[-h, 0]$, where:

$$
\begin{aligned}
& C=A+D_{x} F(0,0,0), E=B+D_{u} F(0,0,0), \\
& G=D+D_{u(t-h)} F(0,0,0)
\end{aligned}
$$

be associated with a semilinear dynamical system (13).

Theorem 4.1. $\boldsymbol{U}_{c}$-local controllability [40]. Suppose that:

H1) $F(0,0,0)=0$,

H2) $U_{c} \subset U$ is a closed and convex cone with vertex at zero,

H3) The associated linear control system (18) is $U_{c}$-globally controllable in $[0, \mathrm{~T}]$.

Then, the semilinear stationary control system $(11)$ is $U_{c}$-locally controllable in $[0, \mathrm{~T}]$.

Corollary. $\boldsymbol{U}_{\boldsymbol{c}}$-global controllability [40]. Suppose that the dynamical system (11) has single input, i.e., $m=1$ and $U_{c}=R^{+}$. Then, the associated linear dynamical control system (18) is $U_{c}$-globally controllable in $[0, T]$, for $T \leq h$ if and only if it is controllable without any constraints, i.e.:

$$
\operatorname{rank}\left[E, C E, C^{2} E, \ldots, C^{2 n-1} E\right]=2 n,
$$

and matrix $C$ has only complex eigenvalues.

Theorem 4.1 is proved using a generalized open mapping theorem.

\subsection{Trajectory controllability of second order semilinear} integro-differential systems. Generally speaking, the trajectory controllability means, that it is possible to steer the dynamical control system from an arbitrary initial state to an arbitrary final state, along a prescribed trajectory, using the set of admissible controls. 
In trajectory controllability, the sufficient condition for semilinear system can be formulated and proved using wellknown Gronwal's inequality [43]. Let $H$ an $U$ be the Hilbert spaces. Consider semilinear integro-differential control system described by the following equation:

$$
\begin{aligned}
& \frac{d^{2}}{d t^{2}} w(t)=A w(t)+B(t, u(t))+ \\
& \quad+F\left(t, w(t), \int_{0}^{t} G(t, s, w(s)) d s\right) \\
& w(0)=w_{0}, \frac{d}{d t} w(0)=w_{1}, t \in[0, T]
\end{aligned}
$$

where:

1. the state $w(t) \in H$ and control $u(t) \in U=L^{2}[0, T]$ for $t \in[0, T]$,

2. the operator $A: H \rightarrow H$ is a linear and not necessarily bounded operator,

3. the maps $B:[0, T] \times U \rightarrow H, G: \triangle \times H \rightarrow H$ and $F:[0, T] \times H \times H \rightarrow H$ are nonlinear operators, where $\triangle=\{(t, s)=[0, T] \times[0, T]: 0 \leq s \leq t \leq T\}$.

Definition 4.4. Trajectory controllability [43]. Let $\tau$ be the set of all functions $z \in L^{2}([0, T], H)$ which are twice continuously differentiable so that $z(0)=w_{0}$ and $\tau_{1}$ be the set of all functions $\dot{z} \in L^{2}([0, T], H)$ such that $\dot{z}(0)=w_{1}\left(\tau_{1} \in \tau\right)$. Control system (20) is said to be trajectory controllable if for any $z \in \tau$ and $\dot{z} \in \tau_{1}$ there exist an $L^{2}$-function $u:[0, T] \rightarrow H$ such that the corresponding solution of equation (20) satisfies $w(\cdot)=z(\cdot)$ a.e.

Definition 4.5. Mild solution [43]. A function $w(t)$ is said to be a mild solution of system (20) if it satisfies the following Volterra integral equation:

$$
\begin{gathered}
x(t)=C(t) w_{0}+S(t) w_{1}+\int_{0}^{t} S(t-s) B(s, u(s) d s+ \\
+\int_{0}^{t} S(t-s) F\left(s, w(s), \int_{0}^{s} G(s, \tau, w(\tau)) d \tau\right) d s \\
t \in[0, T] .
\end{gathered}
$$

where $C(t)$ and $S(t), t \in R$ denotes the strongly continuous cosine and associated sine families of linear bounded operators in the space $H$ generated by the operator $A$, respectively.

Theorem 4.2. Mild solution [43]. Suppose that:

H1) Operator $A$ is the infinitesimal generator of a strongly continuous cosine family $C(t), t \in R$ of bounded linear operators from $X$ into itself and the adjoint operator $A^{*}$ is densely defined i.e., $\overline{\mathscr{D}\left(A^{*}\right)}=X^{*}$,

H2) $B$ and $G$ satisfy the Caratheodory condition, i.e.:,

$B(t, \cdot): U \rightarrow H$ is continuous for $t \in[0, T]$,

$B(\cdot, u):[0, T] \rightarrow H$ is measurable for $u \in U$,

$G(t, s, \cdot): H \rightarrow H$ is continuous for $\forall(t, s) \in \Delta$,

$G(\cdot, \cdot, x): \Delta \rightarrow H$ is measurable for $\forall x \in H$,
H3) $F$ satisfies is Caratheodory condition, i.e. $F(\cdot, x, y)$ is measurable with respect to first argument and $F(t, \cdot, \cdot)$ is continuous with respect to second and third arguments,

H4) $B, G, F$ satisfy the growth conditions:

$\|B(t, u)\|_{H} \leq b_{0}(t)+b_{1}\|u\|_{U} \forall u \in U, t \in[0, T]$,
$\|G(t, s, x)\|_{H} \leq q_{0}(t)+q_{1}\|u\|_{H} \forall t \in[0, T], x \in H$,
$\|F(t, s, x)\|_{H} \leq a_{0}(t)+a_{1}\|x\|_{H}+a_{2}\|y\|_{H}$.

Then, system (20) has a mild solution on $[0, T]$ given by (21).

Theorem 4.3. Trajectory controllability [43]. Suppose hypotheses $(\mathrm{H} 1-\mathrm{H} 4)$ of Theorem 4.2 are satisfied and additionally, the following conditions hold:

H5) $F(t, s, x)$ is Lipschitz continuous with respect to $x$ and $y$, i.e. there exist constants $\alpha_{1}, \alpha_{2} \geq 0$ such that:

$\left\|F\left(t, x_{1}, y_{1}\right)-F\left(t, x_{2}, y_{2}\right)\right\|_{H} \leq a_{0}\left\|x_{1}-x_{2}\right\|_{H}+a_{1}\left\|y_{1}-y_{2}\right\|_{H}$, for $x_{1}, x_{2}, y_{1}, y_{2} \in H, t \in[0, T]$,

H6) $G(t, s, x)$ is Lipschitz continuous with respect to $x$, i.e. there exist constants $\beta>0$ such that:

$\|G(t, s, x)-G(t, s, y)\|_{H} \leq \beta\|x-y\|_{H}, x, y \in H,(t, s) \in \Delta$,

H7) $B$ satisfies monotonicity and coercivity conditions that is:

$$
\langle B(t, u)-B(t, v), u-v\rangle \geq 0 \forall u, v \in U, t \in[0, T]
$$

and

$$
\lim _{\|u\| \rightarrow \infty} \frac{\langle B(t, u), u\rangle}{\|u\|}=\infty .
$$

Then, the nonlinear control system (20) is trajectory controllable.

Proof of Theorem 4.3 is based on theory of monotone operators and tools of cosine operators.

For the special case of (20), i.e. $H=R$ and $U=R$, it is possible to prove stronger result. Consider semilinear finite-dimensional integro-differential control system described by the following semilinear state equation:

$$
\begin{gathered}
\frac{d^{2}}{d t^{2}} x(t)=a(t) x(t)+b(t, u(t))+ \\
f\left(t, x(t), \int_{0}^{t} g(t, s, x(s)) d s\right) \\
x(0)=x_{0}, \frac{d}{d t} x(0)=x_{1}, t \in[0, T], T<\infty .
\end{gathered}
$$

where the state $x(t) \in R$ and control $u(t) \in R$ for $t \in[0, T]$, $a(t) \in L^{1}[0, T]$ and $b:[0, T] \times R \times R \rightarrow R$ for $t \in[0, T]$. Furthermore, $f:[0, T] \times R \times R \rightarrow R$ and $g: \triangle \times R \times R \rightarrow H$ are nonlinear functions satisfying Caratheodory conditions, where $\triangle=\{(t, s)=[0, T] \times[0, T]: 0 \leq s \leq t \leq T\}$.

Definition 4.6. (Trajectory controllability [43]). Control system (22) is said to be trajectory controllable if for any $z \in \tau$ and $\dot{z} \in \tau_{1}$ there exist a control $u \in L^{2}[0, T]$ such that the corresponding solution $x(t)$ of equation (22) satisfies $x(t)=z(t)$

Theorem 4.4. Trajectory controllability [43]. Suppose that:

H1) $b(t, u)$ is continuous,

$\mathrm{H} 2) b(t, u)$ is coercive in second variable, i.e. $b(t, u) \rightarrow \pm \infty$ as $u \rightarrow \pm \infty$, 
H3) the function $f$ is Lipschitz continuous in the second and third variable, uniformly in $t$, i.e. there exist constants $\alpha_{1}, \alpha_{2} \geq 0$ such that:

$$
\left|f\left(t, x_{1}, y_{1}\right)-f\left(t, x_{2}, y_{2}\right)\right| \leq a_{0}\left|x_{1}-x_{2}\right|+a_{1}\left|y_{1}-y_{2}\right|
$$

for $x_{1}, x_{2}, y_{1}, y_{2} \in R, t \in[0, T]$,

H4) the function $g$ is Lipschitz in the third variable uniformly in $(t, s) \in \Delta$, i.e. there exists $\beta>0$ such that:

$$
|g(t, s, x)-g(t, s, y) \leq \beta| x-y \mid, \forall x, y \in R,(t, s) \in \Delta .
$$

Then, the nonlinear control system (22) is trajectory controllable.

Remark 4. The control $u$ obtained in Theorem 4.4 is measurable but may not be continuous. For the continuity of control function $u$ additional assumptions on $b(t, u)$ are required. Control $u(t)$ can be computed directly if the nonlinear function $b(t, u)$ is invertible (43).

4.3. Stochastic systems. Classical control theory is developed for deterministic systems. However, uncertainty is a fundamental characteristic of many real dynamical systems. Moreover, stochastic modelling has been widely used to model the phenomena arising in such branches of science and industry as biology, reliability and risk theory, economics, mechanics, electronics and telecommunications. Therefore, controllability of linear and nonlinear stochastic systems have been a subject of intense research over the last few years [44, 45, 16, 46-49]. The proofs of the controllability results for stochastic systems are based on theorems of the theory of stochastic processes, linearization methods for stochastic dynamical systems, theory of semi-groups of linear operators, Banach, Schauder, Schaefer, or Nussbaum fixed-point theorems and on the so-called generalized open mapping theorem.

4.3.1. Approximate controllability of a second order neutral stochastic differential equations with state dependent delay. Consider dynamical system described by the following partial neural stochastic differential equation with state delay:

$$
\begin{aligned}
& d\left(\frac{d}{d t} x(t)+g\left(t, x_{t}\right)\right)= \\
& =\left[A x(t)+f\left(t, x_{\rho\left(t, x_{t}\right)}\right)+B u(t)\right] d t+G\left(t, x_{t}\right) d W(t), \\
& \text { a.e. on } t \in[0, T] \quad x(0)=\phi \in \mathfrak{B}, \frac{d}{d t} x(0)=\psi \in X
\end{aligned}
$$

where:

1. $A: \mathscr{D}(A) \subset X \rightarrow X$ is the infinitesimal generator of a strongly continuous cosine family $\{C(t): t \in R\}$ of bounded linear operators on a Hilbert space $X$,

2. $\{S(t): t \in R\}$ is the strongly continuous sine family associated to a strongly continuous cosine family $\{C(t): t \in R\}$ defined as $S(t) x=\int_{0}^{t} C(s) x d s, x \in X, t \in R$,

3. $(\Omega, \mathcal{F}, P)$ is a complete probability space with probability measure $P$ on $\Omega$ equipped with a normal filtration $\mathcal{F}, t \geq 0$.
It is endowed with complete family of right continuous increasing sub $\sigma$-algebras $\left\{\mathcal{F}_{t}, t \in[0, T]\right\}$ such that $\mathcal{F}_{t} \subset \mathcal{F}$,

4. The stochastic process is a collection of random variables $S=\{x(t, w): \Omega \rightarrow X: t \in[0, T]\}$ and the control $u(t) \in L_{2}^{\mathcal{F}}([0, T], U)$, where $X$ and $U$ are separable Hilbert spaces and $d$ is the stochastic differentiation.

5. The history valued function $x_{t}:(-\infty, 0] \rightarrow X, x_{t}(\theta)=x(t$ $+\theta$ ) belongs to some abstract phase space $\mathfrak{B}$ defined axiomatically [21],

6. $B$ is a bounded linear operator on a Hilbert space $U$ into $X$,

7. $\rho:[0, T] \times \mathfrak{B} \rightarrow(-\infty, T]$ is continuous function, which describes delay at time $t$ dependent on state,

8. $K$ is a separable Hilbert space and $\{W(t)\}_{t \geq 0}$ is a given $K$-valued Brownian motion or Wiener process with finite trace nuclear covariance operator $Q>0$ given explicitly by:

$$
W(t)=\sum_{n=1}^{\infty} \sqrt{\lambda_{n}} \beta_{n}(t) e_{n}(t), t \geq 0
$$

where $\beta_{n}(t)(n=1,2, \ldots)$ is a sequence of real-valued one-dimensional standard Brownian motions mutually independent over $(\Omega, \mathcal{F}, P), \lambda_{n} \geq 0(n=1,2, \ldots)$ are non-negative real numbers and $\left\{e_{n}\right\}(n=1,2, \ldots)$ is a complete orthonormal basis in $K, Q \in \mathscr{L}(K)$ is an operator defined by $Q e_{n}=\lambda e_{n}$ with finite trace $\operatorname{Tr}(Q)=\sum_{n=1}^{\infty} \lambda_{n} \leq \infty$,

9. The functions $f, g:[0, T] \times \mathfrak{B} \rightarrow X$ are measurable mappings in $X$ norm and $G:[0, T] \times \mathfrak{B} \rightarrow L_{Q}(K, X)$ is measurable mapping in $L_{Q}([0, T], X)$ norm. $L_{Q}([0, T], X)$ is the space of all Q-Hilbert Schmidt operators from $K$ into $X$, i.e. $\forall \phi \in L_{Q}([0, T], X)$ :

$$
\|\phi\|_{Q}^{2}=\operatorname{Tr}\left(\phi Q \phi^{*}\right)=\sum_{n=1}^{\infty}\left\|\sqrt{\lambda_{n}} \phi e_{n}\right\|^{2}<\infty,
$$

10. $\phi(t)$ is $\mathfrak{B}$-valued random variable independent of Brownian motion $W(t)$ with finite second moment. Also $\psi(t)$ is an $X$-valued $\mathcal{F}_{t}$ measurable function.

Associate with semilinear control system (23) a linear control system given by:

$$
\begin{gathered}
\frac{d^{2}}{d t^{2}} x(t)=A x(t)+B u(t), t \in[0, T] \\
x(0)=x_{0}, \frac{d}{d t} x(0)=x_{1} .
\end{gathered}
$$

Let $E$ denote expectation defined by $E(h)=\int_{\Omega} h(\omega) d P$. Let $L_{2}(\Omega, \mathcal{F}, P ; X) \equiv L_{2}(\Omega, X)$ be the Banach space of all strongly measurable, square integrable, $\mathrm{X}$-valued random variables equipped with the norm $\|x(\cdot)\|_{L_{2}}^{2}=E\|x(\cdot)\|_{X}^{2}$. $C\left((-\infty, T], L_{2}(\Omega, X)\right)$ denotes the Banach space of all cotinuous maps from $(-\infty, T]$ into $L_{2}(\Omega, X)$ which satisfies $\sup _{t \in(-\infty, T]} E\|x(t)\|^{2}<\infty$ and $L_{2}^{0}(\Omega, X)=\left\{f \in L_{2}(\Omega, X): f\right.$ is $\mathcal{F}_{0}$-measurable .

Definition 4.7. Mild solution. An $\mathcal{F}_{t}$-adopted process $x:(-\infty, T]$ $\rightarrow X$ is a mild solution to the system (23) if $x_{0}=\phi, \frac{d}{d t} x(0)=\psi$, 
$x(\cdot) \in C^{1}\left([0, T], L^{2}(\Omega, X)\right)$, the functions $f(s, x)_{\rho_{\left(s, x_{s}\right)}}, G\left(s, x_{s}\right)$ and $g\left(s, x_{s}\right)$ are integrable and the integral equation is satisfied:

$$
\begin{aligned}
x(t) & =C(t) \phi(0)+S(t)[\psi+g(0, \phi)] \\
& -\int_{0}^{t} C(t-s) g\left(s, x_{s}\right) d s \\
& +\int_{0}^{t} S(t-s)\left[f\left(s, x_{\rho\left(s, x_{s}\right)}+B u(s)\right] d s\right. \\
& +\int_{0}^{t} S(t-s) G\left(s, x_{s}\right) d W(s), t \in[0, T] .
\end{aligned}
$$

Below the reachable set for unconstrained values of admissible controls is defined.

Definition 4.8. Reachable set. The set given by $R_{T}(f)=\{x(T)$ $\in X: x$ is a mild solution of (23) given by (27); it is called reachable set of the system (23) for some $T>0 . R_{T}(0)$, and it is the reachable set of the corresponding linear control system (26).

Definition 4.9. Approximate controllability. The system (23) is said to be approximately controllable if $R_{T}(f)$ is dense in $X$. The corresponding linear system (26) is approximately controllable if $R_{T}(0)$ is dense in $X$.

Theorem 4.5. Mild solution [50]. Suppose that:

H1) The function $t \rightarrow \phi_{t}$ is continuous from $\mathbb{R}\left(\rho^{-}\right)=\{\rho(s, \psi)$ $: \rho(s, \psi) \leq 0\}$ into $\mathfrak{B}$ and there exists a continuous bounded function $J^{\phi}: \mathbb{R}\left(\rho^{-}\right) \rightarrow(0, \infty)$ such that $\left\|\phi_{t}\right\|_{\mathfrak{B}} \leq J^{\phi}(t)\|\phi\|_{\mathfrak{B}}$ for every $t \in \mathbb{R}\left(\rho_{-}\right)$,

H2) $f:[0, T] \times \mathfrak{B} \rightarrow X$ satisfies the following:

(i) For every $x:(-\infty, T] \rightarrow X, x_{0} \in \mathfrak{B}$ and $\left.x\right|_{[0, T]} \in P C$, the function $f(\cdot, \psi):[0, T] \rightarrow X$ is strongly measurable for every $\psi \in \mathfrak{B}$ and $f(\cdot, t)$ is continuous for a.e. $t \in[0, T]$,

(ii) There exists an integrable function $\alpha:[0, T] \rightarrow[0,+\infty]$ and the monotone continuous nondecreasing function $\Upsilon_{f}:[0,+\infty) \rightarrow[0,+\infty)$ such that $\|f(t, v)\| \leq \alpha(t)$ $\Upsilon_{f}\left(\|v\|_{\mathfrak{B}}\right) \forall t \in[0, T]$ and $v \in \mathfrak{B}$,

H3) The function $G$ satisfies the following conditions:

(i) For almost all $t \in[0, T]$ the function $G(t, \cdot)$ : $\mathfrak{B} \rightarrow L_{Q}(K, X)$ is continuous. For all $z \in \mathfrak{B}$ the function $G(\cdot, z):[0, T] \rightarrow L_{Q}(K, X)$ is strongly $\mathcal{F}_{t}$ measurable,

(ii) There exists an integrable function $\alpha_{G}:[0, T] \rightarrow[0, \infty]$ and a monotone continuous nondecreasing function $\Upsilon_{G}:[0,+\infty) \rightarrow[0,+\infty)$ such that:

$$
\|G(t, z)\|_{Q}^{2} \leq \alpha_{G}(t) \Upsilon_{G}\left(\|z\|_{\mathfrak{B}}^{2}\right),
$$

H4) The function $g(\cdot)$ is continuous $\forall(t, v) \in[0, T] \times \mathfrak{B}$ and $g(t, \cdot)$ is Lipschitz continuous such that there exists a positive constant $L_{g}$ such that:

$$
\begin{gathered}
\left\|g\left(t, v_{1}\right)-g\left(t, v_{2}\right)\right\| \leq L_{g}\left\|v_{1}-v_{2}\right\|_{\mathfrak{B}}, \\
\left(t, v_{i}\right) \in[0, T] \times \mathfrak{B},(i=1,2)
\end{gathered}
$$

H5) There exists a function $H:[0, \infty] \times[0, \infty] \rightarrow[0, \infty]$ which is locally integrable in $t . H$ is a continuous, monotone, nondecreasing in second variable and $H(t, 0) \equiv 0$ and:
$E\left(\left\|f\left(t, m_{1}\right)-f\left(t, m_{2}\right)\right\|^{2}\right)+E\left(\left\|G\left(t, m_{1}\right)-G\left(t, m_{2}\right)\right\|^{2}\right) \leq$ $\left.\leq H\left(t, \| m_{1}-m_{2}\right) \|^{2}\right)$, for all $t \in[0, T]$ and $m_{1}, m_{2} \in$ $\in L_{2}(\Omega, \mathcal{F}, P)$

H6) $\tilde{R}+(\tilde{N} a 6+6 \tilde{N} \operatorname{Tr}(Q)) \int_{0}^{a} \alpha(s) d s \lim _{r \rightarrow \infty} \sup \frac{\gamma(r)}{r} \leq 1$, where

$\tilde{R}=12 N a^{2} K_{a}^{2} L_{g},\|C(t)\| \leq N$ and $\|S(t)\| \leq \tilde{N}$ for every $t \in[0, a]$ and $K_{a}=\sup _{t \in[0, T]} K(t)$.

Then the initial value problem (23) has at least one mild solution.

Proof of Theorem 4.5 is based on the measure of non-compactness.

Theorem 4.6. Approximate controllability [50]. Assume that the associated linear control system (26) is approximately controllable on $[0, T]$, the hypotheses $(\mathrm{H} 1-\mathrm{H} 4)$ of Theorem 4.5 are satisfied and additionally, the following conditions hold:

H7) the functions $f, \mathrm{~g}:[0, T] \times \mathfrak{B} \rightarrow X$ are continuous for $t \in[0, T]$ and $\forall z_{1}, z_{2} \in L_{2}([0, T], \mathfrak{B})$ and there exist constants $L_{f}, L_{g}>0$ such that:

$$
\begin{aligned}
\left\|f\left(t, z_{1}\right)-f\left(t, z_{2}\right)\right\| & \leq L_{f}\left\|z_{1}-z_{2}\right\|_{\mathfrak{B}}, \\
\left\|g\left(t, z_{1}\right)-g\left(t, z_{2}\right)\right\| & \leq L_{g}\left\|z_{1}-z_{2}\right\|_{\mathfrak{B}},
\end{aligned}
$$

H8) the function $G:[0, T] \times \mathfrak{B} \rightarrow L_{Q}(K, X)$ is Lipschitz continuous with constant $L_{g}>0$ such that:

$$
\left\|G\left(t, z_{1}\right)-G\left(t, z_{2}\right)\right\| \leq L_{G}\left\|z_{1}-z_{2}\right\|_{\mathfrak{B}}
$$

H9) the space $L^{2}([0, T], X)=\mathfrak{R}_{i}+\overline{R(B)}(i=1,2)$ and $L^{2}([0, T]$, $\left.L_{Q}(K, X)\right)=\mathfrak{R}_{3}+\overline{R(B)}$ where $\mathfrak{R}_{i}=\operatorname{ker}\left(\Lambda_{i}\right)(i=1,2,3)$ and bounded operators $\Lambda_{i}(i=1,2,3)$ are defined as follows:

$$
\begin{gathered}
\Lambda_{1} x(t)=\int_{0}^{T} S(t-s) x(s) d s, \\
\Lambda_{2} x(t)=\int_{0}^{T} C(t-s) x(s) d s, \\
\Lambda_{3} x(t)=\int_{0}^{T} S(t-s) x(s) d W(s) .
\end{gathered}
$$

Then, the semilinear control system with state dependent delay (23) is approximately controllable on $[0, T]$.

4.3.2. Approximate controllability of second order semilinear stochastic systems with variable delay in control and nonlocal conditions. Consider dynamical system described by the following partial neural stochastic semilinear differential state equation with variable delay in control:

$$
\begin{aligned}
& d\left(\frac{d}{d t} x(t)\right)=\left[A(t) x(t)+B_{1} u(t)+B_{2} u(h(t))+\right. \\
& +f(t, x(t))] d t+\sigma(t, x(t) d W(t), t \in[0, T] \\
& x(0)=x_{0}+g(x), \dot{x}(0)=x_{1}+g_{1}(x) \\
& \text { and } u(t)=0 \text { for } t \in[h(0), 0]
\end{aligned}
$$


where:

1. $X, U, K$ are separable Hilbert spaces,

2. $A: \mathscr{D}(A) \subset X \rightarrow X$ is closed, linear and densely defined on $X$ and is the infinitesimal generator of a strongly continuous cosine family $\{C(t): t \in R\}$ of bounded linear operators on a Hilbert space $X$,

3. $\{S(t): t \in R\}$ is the strongly continuous sine family associated to a strongly continuous cosine family $\{C(t): t \in R\}$ defined as $S(t) x=\int_{0}^{t} C(s) x d s, x \in X, t \in R$,

4. $B_{1}, B_{2}$ are bounded linear operators from the $U$ into $X$,

5. $(\Omega, \mathcal{F}, P)$ is a complete probability space with probability measure $P$ on $\Omega$ equipped with a normal filtration $\mathcal{F}_{t}, t \geq 0$. It is endowed with complete family of right continuous increasing sub $\sigma$-algebras $\left\{\mathcal{F}_{t}, t \in[0, T]\right\}$ such that $\mathcal{F}_{t} \subset \mathcal{F}$,

6. $\{W(t)\}_{t \geq 0}$ is a $K$-valued $Q$-Wiener process on $(\Omega, \mathcal{F}, P)$ given explicitly by (24) with covariance operator $Q$ with finite trace $\operatorname{Tr}(Q)<\infty$,

7. $\mathcal{F}_{t}=\mathcal{F}_{t}^{W}$, where $\mathcal{F}_{t}^{W}$ is the $\sigma$-algebra generated by $W$,

8. $L_{2}^{0}=L_{2}\left(Q^{1 / 2} K ; X\right)$ is the space of all Hilbert-Schmidt operator from $Q^{1 / 2} K$ to $X$ with inner product $\langle\psi, \pi\rangle_{L_{2}^{0}}=\operatorname{Tr}\left(\psi Q \pi^{*}\right)$,

9. $L^{p}\left(\mathcal{F}_{t}, X\right)$ is the Banach space of all $\mathcal{F}_{t}$-measurable $p^{t h}$ power integrable random variables with values in Hilbert space $X, L_{\mathcal{F}}^{p}([0, T], X)$ is the space of all $\mathcal{F}_{t}$ adopted, $X$-valued stochastic processes on $[0, T] \times \Omega$,

10. $C\left([0, T], L^{p}(\mathcal{F}, X)\right)$ is the Banach space of continuous maps from $[0, T]$ into $L^{p}(\mathcal{F}, X)$ satisfying condition $\sup _{t \in[0, T]} E \| x\left(t \|^{p}<\infty\right.$, where $E$ denotes the expectation,

11. $H_{2}=C_{p}([0, T], X)$ is the closed space of $C\left([0, T], L^{p}(\mathcal{F}, X)\right)$ consisting of measurable and $\mathcal{F}_{t}$-adopted, $X$ valued processes $\phi \in C\left([0, a], L^{p}(\mathcal{F}, X)\right)$ endowed with the norm:

$$
\|\phi\|_{H_{2}}=\left(\sup _{t \in[0, T]} E\|\phi\|_{X}^{p}\right)^{1 / p}
$$

12. $f:[0, T] \times X \rightarrow X$ and $\sigma:[0, T] \times X \rightarrow L_{2}^{0}$ are nonlinear suitable functions,

13. $x_{0}$ and $x_{1}$ are $\mathcal{F}_{0}$-measurable $X$ valued random variables independent of $W$,

14. $g$ and $g_{1}$ are continuous functions from $C([0, T], X) \rightarrow X$,

15. $h(t)=t-h_{1}(t)$ is continuous, differentiable and strictly increasing on $[0, T]$ and $h_{1}(t)>0$. Function $r(t)=t+h_{1}(t)$ with $h_{1}(t)$ satisfying $r\left(h_{1}(t)\right)=t$. In fact, $r(t)$ is inverse function of $h(t)$,

16. $u \in U_{a d}$, where $U_{a d}$ is the set of admissible controls $U_{a d}=L_{\mathfrak{F}}^{p}$ $([0, T], X)$.

Definition 4.10. (Mild solution). A stochastic process $x \in \mathrm{H}_{2}$ is a mild solution of (28) if for each $u \in L^{2}([0, T], U)$ it satisfies the following integral equation:

$$
\begin{aligned}
x(t, u) & =C(t)\left(x_{0}+g(x)\right)+S(t)\left(x_{1}+g_{1}(x)\right)+ \\
& +\int_{0}^{t} S(t-s)\left[B_{1}(s) u(s)+f(s, x(s))\right] d s \\
& +\int_{0}^{t} S(t-s) B_{2}(s) u(h(s)) d s \\
& +\int_{0}^{t} S(t-s) \sigma(s, x(s) d W(s), t \in[0, T] .
\end{aligned}
$$

Definition 4.11. (Approximate controllability). The stochastic dynamical system (28) is approximately controllable on $[0, T]$ if $\overline{R(T)}=L^{p}\left(\mathcal{F}_{T}, X\right)$, where $R(T)=\left\{x(T, u): u \in U_{a d}\right\}$.

Theorem 4.7. Mild solution [51]. Suppose that:

H1) the functions $f:[0, T] \times X \rightarrow X$ and $\sigma:[0, T] \times X \rightarrow L_{2}^{0}$ satisfy linear growth and Lipschitz conditions, i.e., there exist positive constants $N_{1}, N_{2}, K_{1}, K_{2}$ such that:

$$
\begin{gathered}
\|f(t, x)-f(t, y)\|^{p} \leq N_{1}\|x-y\|^{p}, \\
\|f(t, x)\|^{p} \leq N_{2}\left(1+\|x\|^{p}\right), \\
\|\sigma(t, x)-\sigma(t, y)\|_{L_{2}^{0}}^{p} \leq K_{1}\|x-y\|^{p}, \\
\|\sigma(t, x)\|_{L_{2}^{0}}^{p} \leq K_{2}\left(1+\|x\|^{p}\right),
\end{gathered}
$$

H2) the functions $g$ and $g_{1}$ are continuous and there exist some positive constants $M_{g}$ and $M_{g 1}$ such that:

$$
\begin{gathered}
\|g(x)-g(y)\|^{p} \leq M_{g}\|x-y\|^{p}, \\
\|g(x)\|^{p} \leq M_{g}\left(1+\|x\|^{p}\right), \\
\left\|g_{1}(x)-g_{1}(y)\right\|^{p} \leq M_{g_{1}}\|x-y\|^{p}, \\
\left\|g_{1}(x)\right\|^{p} \leq M_{g_{1}}\left(1+\|x\|^{p}\right),
\end{gathered}
$$

for all $x, y \in C([0, T], X)$,

H3) for each $0 \leq t \leq T$, the operator $\alpha\left(\alpha I+\Psi_{t}^{T}\right)^{-1} \rightarrow 0$ in the strong operator topology as $\alpha \rightarrow 0^{+}$where the operator $\Psi_{t}^{T}$ is defined as:

$$
\begin{aligned}
\Psi_{t}^{T}=\int_{t}^{h(T)}\left[r^{\prime}(s) S(T-r(s)) \widetilde{B}(r(s))\right. \\
\left.S^{*}(T-r(s)) r^{\prime}(s)\right] d s+ \\
+\int_{t}^{h(T)}\left[S(T-s) B_{1}(s) B_{1}^{*}(s) S^{*}(T-s)\right] d s+ \\
+\int_{h(T)}^{T}\left[S(T-s) B_{1}(s) B_{1}^{*}(s) S^{*}(T-s)\right] d s,
\end{aligned}
$$

where $\tilde{B}(r(s))=B_{2}(r(s)) B_{2}^{*}(r(s))$.

Then, the system (28) has a mild solution on $[0, T]$.

Theorem 4.8. Approximate controllability [51]. If the hypotheses $(\mathrm{H} 1-\mathrm{H} 3)$ of the theorem 4.7 are satisfied and $\{S(t): t \geq 0\}$ is compact and functions $f$ and $\sigma$ are uniformly bounded, the the system (28) is approximately controllable on $[0, T]$.

Proof of Theorem 4.8 is based on the Banach fixed-point theorem.

4.3.3. Approximate controllability of second order neural stochastic differential equations with infinite delay and Poisson jumps. Consider second order neural stochastic dif- 
ferential equation with infinite delay in the state variable and Poisson jumps:

$$
\begin{gathered}
d\left[\frac{d}{d t} x(t)-f\left(t, x_{t}\right)\right]= \\
{[A x(t)+B u(t)] d t+g\left(t, x_{t}\right) d W(t)} \\
+\int_{Z} h\left(t, x_{t}, \eta\right) \tilde{N}(d t, d \eta), t \in[0, T] \\
x(0)=\phi \in \mathfrak{B}, \frac{d}{d t} x(0)=\xi
\end{gathered}
$$

where:

1. state variable $x(\cdot)$ takes values in a Hilbert space $H$ with inner product $\langle\cdot, \cdot\rangle$ and norm $\|\cdot, \cdot\|$, and the control $u(\cdot)$ in given in $L_{2}([0, T], U)$, a Banach space of admissible controls with $U$ as a Banach space,

2. $K$ is a separable Hilbert space and $\{W(t)\}_{T \geq 0}$ is a $K$-valued $Q$-Wiener process on $(\Omega, \mathcal{F}, P)$ given explicitly by $(24)$ with covariance operator $Q$ with finite trace $\operatorname{Tr}(Q)<\infty$,

3. $q=(q(t)), t \in D_{q}$ is stationary $\mathcal{F}_{t}$-Poisson point process with a characteristic measure $\lambda . N(d t, d \eta)$ is the Poisson counting measure associated with $q$, so $N(t, Z)=$ $=\sum_{s \in D_{q}, s \leq t} I_{z}=(q(s))$ with measurable set $Z \in \bar{B}(Y-\{0\})$, which denotes the Borel $\sigma$-field of $Y-\{0\}$,

4. $\tilde{N}(d t, d \eta)=N(d t, d \eta)=d t \lambda(d \eta)$ is the compensated Poisson measure that is independent of $W(t)$,

5. $p^{2}([0, T] \times Z, H)$ is the space of all predictable mappings $\chi:[0, T] \rightarrow H$ for which $\int_{0}^{T} \int_{Z} E\left\|_{\chi}\right\|_{H}^{2} d t \lambda(d \eta)<\infty$, $\int_{0}^{T} \int_{Z} \chi(t, \eta) \tilde{N}(d t, d \eta)$ is a centred square integrable martingale,

6. the history valued function $x_{t}:(-\infty, 0] \rightarrow X, x_{t}(\theta)=x(t+\theta)$ belongs to some abstract phase space $\mathfrak{B}$ defined axiomatically [21],

7. the functions $f:[0, T] \times \mathfrak{B} \rightarrow H, g:[0, T] \times \mathfrak{B} \rightarrow L_{Q}(K, H)$, and $h:[0, T] \times \mathfrak{B} \times Z \rightarrow H$ are nonlinear functions, where $L_{Q}([0, T], H)$ is the space of all Q-Hilbert Schmidt operators from $K$ into $H$, i.e. such that (25) holds true,

8. the initial data $\psi=\{\phi(t):-\infty<t \leq 0\}$ is $\mathcal{F}_{0}$-measurable $\mathfrak{B}$-valued stochastic process independent of Brownian motion $W(t)$ and Poisson point process $q$ with finite second moment. Also $\xi(t)$ is an $\mathcal{F}_{t}$-measurable $H$-valued $\mathcal{F}_{t}$ random variable independent of $W(t)$ and Poisson point process $q$ with finite second moment,

9. $(\Omega, \mathcal{F}, P)$ is a complete probability space with probability measure $P$ on $\Omega$ equipped with a normal filtration $\mathcal{F}_{t}, t \geq 0$. It is endowed with complete family of right continuous increasing sub $\sigma$-algebras $\left\{\mathcal{F}_{t}, t \in[0, T]\right\}$ such that $\mathcal{F}_{t} \subset \mathcal{F}$,

10. a $\mathrm{H}$-valued random variable is an $\mathcal{F}$-measurable function $x(t): \Omega \rightarrow H$, and a collection of random variable $S=\{x(t$, $\left.\omega): \Omega \rightarrow H:\left.\right|_{t \in[0, T]}\right\}$ is a stochastic process. The dependence on $\omega \in \Omega$ is suppressed and $x(t)$ is written instead of $x(t, \omega)$,

11. $A: \mathscr{D}(A) \subset X \rightarrow X$ is the infinitesimal generator of a strongly continuous cosine family $\{C(t): t \in R\}$ of bounded linear operators on a Hilbert space $X$,
12. $\{S(t): t \in R\}$ is the strongly continuous sine family associated to a strongly continuous cosine family $\{C(t): t \in R\}$ defined as $S(t) x=\int_{0}^{t} C(s) x d s, x \in X, t \in R$,

13. $B$ is a bounded linear operator from $L_{2}([0, T], U)$ to $L_{2}([0, T], H)$.

Definition 4.12. (Mild solution). A stochastic process $x_{t}(v)$ is a mild solution of (30) if for each $u \in L^{2}([0, T], H)$ it satisfies the following integral equation:

$$
\begin{aligned}
x_{t}(v) & =C(t) \phi(0)+S(t)(\xi-f(0, \phi)) \\
& +\int_{0}^{t} C(t-s) f\left(s, x_{s}(v)\right) d s \\
& +\int_{0}^{t} S(t-s) v(s) d s+\int_{0}^{t} S(t-s) g\left(s, x_{s}(v)\right) d W(s) \\
& +\int_{0}^{t} \int_{Z} S(t-s) h\left(s, x_{s}(v), \eta\right) \tilde{N}(d s, d \eta), t \in[0, T] .
\end{aligned}
$$

Definition 4.13. (Approximate controllability). The stochastic dynamical system (30) is approximately controllable on $[0, T]$ if $\overline{R_{T}(g, h)}=L_{2}(\Omega, H)$, where $R_{T}(g, h)=\left\{x_{T}(B u): u \in L_{2}([0, T]\right.$, $H)\}, x_{T}(B u)$ is a mild solution of (30) given by (31) with $v=B u$.

Theorem 4.9. Approximate controllability [52]. Suppose that: H1) the nonlinear functions $f, g$ satisfy Lipschitz condition i.e., there exist positive constants $M_{f}, M_{g}$ such that:

$$
\begin{gathered}
\left\|f\left(t, x_{1}\right)-f\left(t, x_{2}\right)\right\|^{2} \leq M_{f}\left\|x_{1}-x_{2}\right\|_{\mathfrak{B}}^{2}, \\
\left\|g\left(t, x_{1}\right)-g\left(t, x_{2}\right)\right\|^{2} \leq M_{g}\left\|x_{1}-x_{2}\right\|_{\mathfrak{B}}^{2},
\end{gathered}
$$

for all $x_{1}, x_{2} \in \mathfrak{B}$ and $t \in[0, T]$

H2) the nonlinear function $h$ satisfies Lipschitz condition and there exist positive constants $M_{h}, L_{h}$ such that:

$\int_{Z}\left\|h\left(t, x_{1}, \eta\right)-h\left(t, x_{2}, \eta\right)\right\|^{2} \lambda(d \eta) \leq M_{h}\left\|x_{1}-x_{2}\right\|_{\mathfrak{B}}^{2}$,
$\int_{Z}\left\|h\left(t, x_{1}, \eta\right)-h\left(t, x_{2}, \eta\right)\right\|^{4} \lambda(d \eta) \leq M_{h}\left\|x_{1}-x_{2}\right\|_{\mathfrak{B}}^{4}$,

for all $x_{1}, x_{2} \in \mathfrak{B}$ and $t \in[0, T]$

H3) for any given $\varepsilon>0$ and $p(\cdot) \in L_{2}([0, T], H)$, there exists some $u(\cdot) \in L_{2}([0, T], U)$ such that:

i) $\|\Phi p,-\Phi B u\|_{H}<\varepsilon$, where the continuous linear operator $\Phi$ from $L_{2}([0, T], H)$ to $L_{2}(\Omega, H)$ is defined as:

$$
\Phi p=\int_{0}^{T} S(T-s) p(s) d s, \text { for } p \in L_{2}([0, T], H),
$$

ii) $\|B u(\cdot)\|_{L_{2}([0, T], H)} \leq q_{1}\|p(\cdot)\|_{L_{2}([0, T], H)}$, where $q_{1}$ is a positive constant dependent of $p(\cdot)$,

iii) the constant $q_{1}$ satisfies:

$$
\begin{aligned}
& z \exp \left\{4 \overline { K } _ { 3 } b \left\{b M_{C}^{2} M_{f}+\operatorname{Tr}(Q) M_{S}^{2} M_{g}+\right.\right. \\
& \left.\left.+b M_{S}^{2}\left(M_{h}+\sqrt{L_{h}}\right)\right\}\right\}<1 \text {, } \\
& \text { where } z=8 q_{1} b^{2} M_{s}^{2} \bar{K}_{3}\left(\operatorname{Tr}(Q) M_{g}+b\left(M_{h}+\sqrt{L_{h}}\right)\right. \text {. }
\end{aligned}
$$

Then, the system (30) is approximately controllable on $[0, T]$. 
Proof of Theorem 4.9 is based on semigroup theory and stochastic analysis techniques.

4.4. Controllability of Duffing equation. An increasing interest in studying problems related to controllability of chaotic nonlinear systems has been observed in recent years. In several papers Duffing equation is treated as a benchmark chaotic system [53, 54]. It was introduced in 1918 by Duffing as a model of nonlinear oscillator with cubic stiffness term to describe the hardening spring effect observed in many mechanical systems [55].

Consider the following controllability problem for a feedback control system governed by the equation:

$$
\begin{aligned}
& \frac{d^{2}}{d t^{2}} x(t)+\delta \frac{d}{d t} x(t)+\alpha x(t)+\beta x^{3}(t)= \\
& =f(t, x(t), u(t)), \text { for } t \in[0, T]
\end{aligned}
$$

and functional inclusion:

$$
\begin{aligned}
& \frac{d}{d t} x(t) \in F(t, x(t), u(t)), \text { a.e. on } t \in[0, T], \\
& x(0)=x_{0}, x(T)=x_{1}, u(0)=u_{0},
\end{aligned}
$$

where:

1. the functions $x:[0, T] \rightarrow R$ and $u:[0, T] \rightarrow R$ describe the state of the system and the control, respectively,

2. parameters $\delta, \alpha, \beta$ are given constants $\beta<0$ and $x_{0}, x_{1}, u_{0} \in R$,

3. the continuous function $f:[0, T] \times R \times R \rightarrow R$ characterizes the dynamics of the system,

4. the multimap $F:[0, T] \times R \times R \rightarrow R$ represents the feedback law.

Definition 4.14. (Upper semicontinuous multimap [56]). Let $X, Y$ be the metric spaces and $P(Y)$ the collection of all nonempty subset of $Y$. A multivalued map (multimap) $F: X \rightarrow P(Y)$ is said to be upper semicontinuous, if for every open subset $V \subset Y$ the set

$$
F_{+}^{-1}(V)=\{x \in X: F(x) \subset V\}
$$

is open in $X$.

Definition 4.15. (Solution to the controllability problem [56]). A pair consisting of a function $x \in C^{2}([0, T]$ and an absolutely continuous function $u$ satisfying all relations (32) is said to be a solution to the controllability problem (32).

Theorem 4.1.0. Controllability [56]. Suppose that:

H1) for every $(z, w) \in R \times R$ the multifunction $F(\cdot, z, w):[0, T]$ $\rightarrow K v(R)$ has a measurable selection, where $K v(E)$ denotes the collection of all nonempty, convex, compact subsets of $E$,

H2) for a.e. $t \in[0, T]$ the multimap $F(t, \cdot, \cdot): R \times R \rightarrow K v(R)$ is upper semicontinuous,
H3) the multimap $F$ is uniformly continuous with respect to the second argument in the following sense: for every $\varepsilon>0$ there is $\kappa>0$ such that

$$
F(t, \bar{z}, w) \subset O_{\varepsilon}(F(t, z, w)), \forall(t, w) \in[0, T] \times R
$$

whenever $|\bar{z}-z|<\kappa$,

H4) there exists $L>0$ such that

$$
\begin{gathered}
\|F(t, z, w)\| \leq L(1+|z|+|w|), \\
|f(t, z, w)| \leq L(1+|z|+|w|)
\end{gathered}
$$

for all $(z, w) \in R \times R$ and a.e. $t \in[0, T]$, where

$$
\|F(t, z, w)\|=\max \{|v|: v \in F(t, z, w)\}
$$

Then, the controllability problem (32) has a solution.

To prove Theorem 4.10 the facts from theory of multivalued maps and theory of differential inclusions as well as the method of a priori bounds were used.

4.5. Exact boundary controllability of coupled hyperbolic equations. The last two decades show the increase of interest in the controllability of coupled equations of the same nature. Much has been done in the case of coupled parabolic equations - see [57] and references therein. In terms of hyperbolic equations one can find few results in [58-61]. In the analysis of distributed parameter systems, the method of moments [62, $63]$ is especially effective. The method is based on the properties of exponential families, such as minimality, the Riesz basis property and the $\mathscr{L}$-basis property, all usually in the space $L^{2}(0, T)$. Recent investigations into new classes of distributed systems, such as hybrid or damped, as well as into coupled equations raised new problems in the moment theory. See for example [64, 63].

Let $\Omega$ be an open bounded subset of $\mathbb{R}^{n}$ and nonempty $\omega, \mathscr{O} \subset \Omega, T>0$. Consider the coupled system:

$$
\begin{cases}\frac{\partial^{2}}{\partial t^{2}} y-\triangle y=h_{\omega} & \text { in } Q:=\Omega \times(0, T) \\ y=0 & \text { on } \Sigma:=\partial \Omega \times(0, T), \\ y(x, 0)=y^{0}(x), \frac{\partial}{\partial t} y(x, 0)=y^{1}(x) & \text { in } \Omega\end{cases}
$$

$$
\begin{cases}\frac{\partial^{2}}{\partial t^{2}} q-\Delta q=y 1_{\mathscr{O}} & \text { in } Q \\ q=0 & \text { on } \Sigma \\ q(x, T)=0, \frac{\partial}{\partial t} q(x, T)=0 & \text { in } \Omega .\end{cases}
$$

The problem is to specify the conditions on $\omega, \mathscr{O}$ and $T>0$ to guarantee that for every $\left(y^{0}, y^{1}\right) \in L^{2}(\Omega) \times H^{-1}(\Omega)$ there exists a control $h$ in appropriate space, such that:

$$
q(x, 0)=\frac{\partial}{\partial t} q(x, 0)=0
$$

The authors of [65] analyse the one-dimensional case, namely $\Omega=(0, \pi), T>0, Q=\Omega \times(0, T)$ and $\alpha, \beta \in \mathbb{R}$. The system in which controllability is studied takes the form: 


$$
\begin{cases}\frac{\partial^{2}}{\partial t^{2}} y-\frac{\partial^{2}}{\partial x^{2}} y+\alpha q=0 & \text { in } Q, \\ \frac{\partial^{2}}{\partial t^{2}} q-\frac{\partial^{2}}{\partial x^{2}} q+\beta y=0 & \text { in } Q, \\ y(0, t)=u(t), y(\pi, t)=0 & t \in(0, T), \\ q=0 & \text { on } \Sigma, \\ y(x, 0)=y^{0}(x), \frac{\partial}{\partial t} y(x, 0)=y^{1}(x) & \text { in } \Omega, \\ q(x, 0)=q^{0}, \frac{\partial}{\partial t} q(x, 0)=q^{1} & \text { in } \Omega .\end{cases}
$$

The method used in [65] is based on the transformation of the controllability problem into a moment problem of the form of the initial boundary value problem (IBVP)

$$
\int_{0}^{T} u(t) f_{n}(t) d t=c_{n}, \quad n \in \mathbb{N}
$$

As shown in [63], the problem (36) has a solution $u \in L^{2}(0, T)$ for any $\left\{c_{n}\right\}_{\mathbb{N}} \in L^{2}(\mathbb{R})$ if and only if the family $\mathscr{F}=\left\{f_{n}\right\}_{n \in \mathbb{N}}$ forms an $\mathscr{L}$-basis in $L^{2}(0, T)$. The family $\mathscr{F}$ has different forms in the case $\alpha=0, \alpha \beta>0$ and $\alpha \beta<0$. Authors of [65] prove that in all these cases, when the condition (37) does not hold, the corresponding family $\mathscr{F}$ forms a Riesz basis in $L^{2}(0, T)$ for $T \geq 4 \pi$.

Let $u \in L^{2}(0, T)$ and set $\mathscr{H}_{0}=L^{2}(0, \pi), \mathscr{H}_{1}=H_{0}^{1}(0, \pi)$, $\mathscr{H}_{-1}=H^{-1}(0, \pi), \mathscr{V}=\mathscr{H}_{0} \times \mathscr{H}^{1} \times \mathscr{H}_{-1} \times \mathscr{H}_{0}$, and $\left(y^{0}, q^{0}, y^{1}, q^{1}\right)$ $\in \mathscr{V}$. Let the solution to the $\operatorname{IBVP}(35)$ be $(y, q)=\left(y^{u}(x, t), q^{u}(x, t)\right)$.

Definition 4.16. (Exact controllability of the IBVP). The system described by (35) is called exactly controllable in the time interval $[0, T]$ if for any $\left(y^{0}, q^{0}, y^{1}, q^{1}\right) \in \mathscr{V}$ there is

$\left\{\left(y^{u}(\cdot, T), q^{u}(\cdot, T), y_{t}^{u}(\cdot, T), q_{t}^{u}(\cdot, T)\right): u \in L^{2}(0, T)\right\}=\mathscr{V}$.

Definition 4.17. (Approximate controllability of the IBVP). The system described by (35) is called exactly controllable in the time interval $[0, T]$ if for any $\left(y^{0}, q^{0}, y^{1}, q^{1}\right) \in \mathscr{V}$ the set

$$
\left\{\left(y^{u}(\cdot, T), q^{u}(\cdot, T), y_{t}^{u}(\cdot, T), q_{t}^{u}(\cdot, T)\right): u \in L^{2}(0, T)\right\}=\mathscr{V} .
$$

is dense in $\mathscr{V}$.

The main results of the authors of [65] can be summarized in the following two theorems.

Theorem 4.11. Existence of a solution to IBVP [65]. Let $\left(y^{0}, q^{0}, y^{1}, q^{1}\right) \in \mathscr{V}$. The solution $(y, q)=\left(y^{u}(x, t), q^{u}(x, t)\right)$ of the IBVP (35) exists, is unique and satisfies the inclusion $\left(y^{u}, q^{u}, y_{t}^{u}, q_{t}^{u}\right) \in C([0, T], \mathscr{V})$. This means that for any $t \in[0, T]$, $\left(y^{u}(\cdot, t), q^{u}(\cdot, t),\left(y_{t}^{u}(\cdot, t), q_{t}^{u}(\cdot, t)\right) \in \mathscr{V}\right.$ and is continuous in $t$ in the norm on $\mathscr{V}$.

Theorem 4.12. Exact controllability of IBVP [65]. Suppose $\beta \neq 0$, and consider the equality

$$
n^{2}-\sqrt{\alpha \beta}=m^{2}+\sqrt{\alpha \beta}, \quad m, n \in \mathbb{N} .
$$

(i) If (37) never occurs for $m \neq n$, the system (35) is exactly controllable in the time interval $[0, T], T \geq 4 \pi$.

(ii) If (37) occurs for some $n, m$, the system (35) is not approximately controllable for any $T$.

(iii) The system (35) is not exactly controllable for $T \geq 4 \pi$.

Remark 5. If $\beta=0$, the second equation is decoupled from the first one, where the control occurs. As a result, the system (35) is not approximately controllable for any $T>0$.

The authors prove their results by means of applying the moments method.

4.6. Exact controllability of impulsive systems. Impulsive dynamical systems are characterized by the occurrence of abrupt changes in the state of the system, which occur at certain time instants over a period of negligible duration. The presence of impulse means that the state trajectory does not preserve the basic properties associated with a non-impulsive dynamical systems. The impulses are frequently modelled as jumps in parameters. The impulsive differential and integrodifferential systems has been used in modelling of population dynamics, ecology, biological systems, biotechnology, industrial robotics, pharmacokinetics, optimal control etc. For the general theory of impulsive differential equations, we refer the reader to the references [66-70]. The exemplary applications of impulsive differential systems may be found in [71-73].

Although the body of literature concerning the presence of delay in differential equations is extensive, the impulsive differential equations with delay seem to be rarely considered. Among the first works devoted to this subject are [74, 75], where the investigation of functional differential equations with infinite delay in an abstract admissible phase space is considered. For a detailed discussion on the method of admissible phase space, we refer the reader to [20].

Additionally one may come across the notion of neutral functional differential equations. This class of equations depends on past as well as on present values which involve derivatives with delays as well as the function itself. A good guide to the literature for neutral functional differential equations is [76] with references therein.

The recent publications $[22,77]$ deal with the combinations of the above types of second order systems. The former presents the approach based on evolution system while the latter refers to the integrodifferential system.

If not stated otherwise, a $P C$ space is endowed with the norm $\|x\|_{P C}=\sup _{s \in J}\|x(s)\|$. It is clear that $\left(P C,\|\cdot\|_{P C}\right)$ is a Banach space. In what follows, we put $t_{0}=0, t_{n+1}=T$ and, for $x \in P C$ we denote by $\tilde{x}_{k}$ for $k=1,2, \ldots, m$ the function $\tilde{x}_{k} \in C\left(\left[t_{k}, t_{k+1}\right], X\right)$ given by $\tilde{x}_{k}(t)=x(t)$ for $t \in\left(t_{k}, t_{k+1}\right]$ and $\tilde{x}_{k}\left(t_{k}\right)=\lim _{t \rightarrow t_{k}^{+}} x(t)$.

In impulsive functional differential systems, the map $[\mu, \mu+b] \rightarrow \mathfrak{B}, t \rightarrow x_{t}$ is, in general, discontinuous. For this reason, this property has been omitted from the description of the phase space $\mathfrak{B}$.

Consider the following second order damped impulsive neutral integrodifferential system with infinite delay of the form: 


$$
\begin{gathered}
\frac{d}{d t}\left[x^{\prime}(t)-h\left(t, x_{t}, x^{\prime}(t)\right)\right]=A x(t)+Q x^{\prime}(t)+ \\
+f\left(t, x_{t}, x^{\prime}(t)\right)+\int_{-\infty}^{t} p\left(t, s, x_{s}, x^{\prime}(s)\right) d s+B u(t) \\
x_{0}=\phi, x^{\prime}(0)=\zeta \\
\triangle x\left(t_{k}\right)=I_{k}\left(x_{t_{k}}, x_{t_{k}}^{\prime}\right), k=1,2, \ldots, m, \\
\triangle x^{\prime}\left(t_{k}\right)=J_{k}\left(x_{t_{k}}, x_{t_{k}}^{\prime}\right), k=1,2, \ldots, m,
\end{gathered}
$$

where:

1. $t \in J=[0, T], t \neq t_{k}, \phi \in \mathfrak{B}$ and $\zeta \in X$,

2. the control function $u(\cdot)$ is given in $L^{2}(J, U)$, a Banach space of admissible control functions with $U$ as a Banach space and $B: U \rightarrow X$ as a bounded linear operator,

3. for $t \in J, x_{t}$ represents the function $x_{t}:(-\infty, 0] \rightarrow X$ defined by $x_{t}(\theta)=x_{t}(t+\theta),-\infty<\theta \leq 0$ which belongs to some abstract phase space $\mathfrak{B}$ defined axiomatically,

4. $f: J \times \mathfrak{B} \rightarrow X, I_{k}: \mathfrak{B} \rightarrow X, J_{k}: \mathfrak{B} \rightarrow X$ are appropriate functions specified below,

5. $0<t_{1}<\ldots<t_{n}<T$ are fixed numbers and the symbol $\triangle \xi(t)$ represents the jump of a function $\xi$ at $t$, which is defined by $\triangle \xi(t)=\xi\left(t^{+}\right)-\xi\left(t^{-}\right)$

6. throughout this paragraph the assumption is that $A: \mathscr{D}(A)$ $\subset X \rightarrow X$ generates the cosine family of bounded linear operators $\{C(t)\}_{t \in \mathbb{R}}$ defined on a Banach space $X$.

The mild solution to the system $(38-41)$ is given by the following.

Definition 4.18. (Mild solution of the system (38-41)). A function $x:(-\infty, T] \rightarrow X$ is called a mild solution of the abstract Cauchy problem (38-41), if $x_{0}=\phi \in \mathfrak{B},\left.x\right|_{I} \in P C^{1}$, the impulsive conditions $\triangle x\left(t_{k}\right)=I_{k}\left(x_{t_{k}}, x_{t_{k}}^{\prime}\right), \triangle x^{\prime}\left(t_{k}\right)=J_{k}\left(x_{t_{k}}, x_{t_{k}}^{\prime}\right)$, $k=1,2, \ldots, m$, are satisfied and the following integral equation:

$$
\begin{aligned}
x(t) & =C(t) \phi(0)+S(t)[\zeta-h(o, \phi, \zeta)] \\
& +\int_{0}^{t} C(t-s) h\left(s, x_{s}, x^{\prime}(s)\right) d s \\
& +\int_{0}^{t} S(t-s)\left[Q x^{\prime}(s)+B u(s)+f\left(s, x_{s}, x^{\prime}(s)\right)\right. \\
& \left.+\int_{0}^{s} p\left(s, \tau, x_{\tau}, x^{\prime}(\tau)\right) d \tau\right] d s \\
& +\int_{0}^{t} S(t-s)\left[\int_{-\infty}^{0} p(s, \tau, \phi, \zeta) d \tau\right] d s \\
& +\sum_{t_{k}<t} C\left(t-t_{k}\right) I_{k}\left(x_{t_{k}}, x_{t_{k}}^{\prime}\right) \\
& +\sum_{t_{k}<t} S\left(t-t_{k}\right) J_{k}\left(x_{t_{k}}, x_{t_{k}}^{\prime}\right), t \in J
\end{aligned}
$$

is verified.
In the above definition:

1. $\{S(t)\}_{t \in \mathbb{R}}$ is the associated sine function family, defined by $S(t) x=f_{0}^{t} C(s) x d s$ for $x \in X$ and $t \in \mathbb{R}$

2. $\tilde{M}$ and $\tilde{N}$ is a pair of positive constants such that $\|C(t)\| \leq \tilde{M}$ and $\|S(t)\| \leq \tilde{N}$ for every $t \in J$.

The Cauchy problem (38-41) is by far, according to the authors knowledge, the most general form of the impulsive system analysed in the literature with constructive controllability results. It contains the damping ingredient and infinite delay. Its analysis, performed in [77], gives the conditions for exact controllability expressed in the form of the following theorem.

Theorem 4.13. Controllability of impulsive system [77]. Denote by $K_{T}=\sup _{0 \leq t \leq T} K(t)$ and suppose that the eight hypotheses below are satisfied:

H1) The function $f: J \times \mathfrak{B} \times X \rightarrow X$ is continuous and there exist constants $L_{f}>0, \tilde{L}_{f}>0$ such that for all $\psi_{j} \in \mathfrak{B}, \varphi_{j} \in X$, $j=1,2$ we have

$$
\begin{aligned}
& \left\|f\left(t, \psi_{1}, \varphi_{1}\right)-f\left(t, \psi_{2}, \varphi_{2}\right)\right\| \leq L_{f}\left[\left\|\psi_{1}-\psi_{2}\right\|_{\mathfrak{B}}+\right. \\
& \left.+\left\|\varphi_{1}-\varphi_{2}\right\|\right] \text { and } \tilde{L}_{f}=\sup _{t \in J}\|f(t, 0,0)\| .
\end{aligned}
$$

H2) The function $p: J \times J \times \mathfrak{B} \times X \rightarrow X$ is continuous and there exist constants $L_{p}>0, \tilde{L}_{p}>0$ such that for all $\psi_{j} \in \mathfrak{B}$, $\varphi_{j} \in X, j=1,2$ we have

$$
\begin{aligned}
& \left\|p\left(t, s, \psi_{1}, \varphi_{1}\right)-p\left(t, s, \psi_{2}, \varphi_{2}\right)\right\| \leq L_{p}\left[\left\|\psi_{1}-\psi_{2}\right\|_{\mathfrak{B}}+\right. \\
& \left.+\left\|\varphi_{1}-\varphi_{2}\right\|\right] \text { and } \tilde{L}_{p}=\sup _{t \in J}\|p(t, s, 0,0)\| .
\end{aligned}
$$

H3) For each $\phi \in \mathfrak{B}$,

$$
v(t)=\lim _{r \rightarrow \infty} \int_{-r}^{0} p\left(t, s, \phi(s), x^{\prime}(s)\right) d s
$$

exists, is continuous and there exists a positive constant $N_{1}$ such that $\|v(t)\| \leq N_{1}$.

H4) $B$ is a continuous operator from $U$ to $X$ and the linear operator $W: L^{2}(J, U) \rightarrow X$, defined by

$W u=\int_{0}^{T} S(T-s) B u(s) d s$,

has a bounded inverse $W^{-1}$ which takes values in $L^{2}(J, U) /$ ker $W$ and there exist positive constants $M_{1}$ and $M_{2}$ such that $\|B\| \leq M_{1}$ and $\left\|W^{-1}\right\| \leq M_{2}$.

H5) The maps $I_{k}, J_{k}: \mathfrak{B} \times \mathfrak{B} \rightarrow X, k=1,2, \ldots m$ are continuous and there exist constants $L_{j}>0, \tilde{L}_{j}>0, j=1,2$ such that for all $\psi_{j}, \varphi_{j} \in \mathfrak{B}, j=1,2$ we have

$$
\begin{aligned}
& \left\|I_{k}\left(\psi_{1}, \varphi_{1}\right)-I_{k}\left(\psi_{2}, \varphi_{2}\right)\right\| \leq L_{1}\left[\left\|\psi_{1}-\psi_{2}\right\|+\left\|\varphi_{1}-\varphi_{2}\right\|\right] \\
& \left\|J_{k}\left(\psi_{1}, \varphi_{1}\right)-J_{k}\left(\psi_{2}, \varphi_{2}\right)\right\| \leq \tilde{L}_{1}\left[\left\|\psi_{1}-\psi_{2}\right\|+\left\|\varphi_{1}-\varphi_{2}\right\|\right] \\
& \text { and } L_{2}=\left\|I_{k}(0,0)\right\|, \tilde{L}_{2}=\left\|J_{k}(0,0)\right\| .
\end{aligned}
$$

H6) The function $h: J \times \mathfrak{B} \times X \rightarrow X$ is continuous and there exist constants $L_{h}>0, \tilde{L}_{h}>0$ such that for all $\psi_{j} \in \mathfrak{B}$, $\varphi_{j} \in X, j=1,2$ we have 
$\left\|h\left(t, \psi_{1}, \varphi_{1}\right)-h\left(t, \psi_{2}, \varphi_{2}\right)\right\| \leq$

$\leq L_{h}\left[\left\|\psi_{1}-\psi_{2}\right\|_{\mathfrak{B}}+\left\|\varphi_{1}-\varphi_{2}\right\|\right]$

and

$\|h(t, \psi, \varphi)\| \leq L_{h}[\|\psi\|+\|\varphi\|]+\tilde{L}_{h}$

where $\tilde{L}_{h}=\max _{t \in J}\|h(t, 0,0)\|$.

H7) Let

$(\tilde{N}+\tilde{M})\left[\|\zeta\|+L_{h}(\|\phi \mid+\| \zeta \|)+\tilde{L}_{h}\right]+(1+T(\tilde{M}+$ $\left.\left.\tilde{N}_{1}\right)\right)\left[L_{h}\left(\left(1+K_{T}\right) r+c_{1}+\left\|\tilde{\phi}^{\prime}\right\|_{T}\right)+\tilde{L}_{h}\right]+(\tilde{N}+\tilde{M})[\|Q\|(r+$ $\left.\left\|\tilde{\phi}^{\prime}\right\|_{T}\right)+\left(L_{f}+T L_{p}\right)\left(\left(1+K_{T}\right) r+c_{1}+\left\|\tilde{\phi}^{\prime}\right\|_{T}\right)+\tilde{L}_{f}+\tilde{B}_{0}+$ $\left.N_{1}+T \tilde{L}_{p}\right]+\sum_{k=1}^{m}\left(\tilde{M}+\tilde{N}_{1}\right)\left[L_{1}\left(2 K_{T} r+\left\|\tilde{\phi}_{t_{k}}\right\|+\left\|\tilde{\phi}_{t_{k}}^{\prime}\right\|+\right.\right.$ $\left.\left.L_{2}\right)\right]+\sum_{k=1}^{m} \tilde{N}+\tilde{M}\left[\tilde{L}_{1}\left(2 K_{T} r+\left\|\tilde{\phi}_{t_{k}}\right\|+\left\|\tilde{\phi}_{t_{k}}^{\prime}\right\|\right)+\tilde{L}_{2}\right] \leq r$, for some $r>0$.

H8) Let $\rho=K_{T}\left[\gamma\left(T \tilde{M} L_{h}+T \tilde{N}\left(L_{f}+T L_{p}\right)+\sum_{k=1}^{m}\left(\tilde{M} L_{1}+\tilde{N} \tilde{L}_{1}\right)\right)\right.$ $+\left(1+T\left(\tilde{M}+\tilde{N}_{1}\right)\right) L_{h}+T(\tilde{N}+\tilde{M})\left(L_{f}+T L_{p}\right)+$ $\left.\sum_{k=1}^{m}\left(\tilde{M}+\tilde{N}_{1}\right) L_{1}+\sum_{k=1}^{m}(\tilde{M}+\tilde{N}) \tilde{L}_{1}\right]$

and

$\tilde{\rho}=\gamma\left(T \tilde{M} L_{h}+T \tilde{N}\left(\|Q\|+L_{f}+T L_{p}\right)+K_{T} \sum_{k=1}^{m}\left(\tilde{M} L_{1}+\right.\right.$ $\left.\left.\tilde{N} \tilde{L}_{1}\right)\right)+\left(1+T\left(\tilde{M}+\tilde{N}_{1}\right)\right) L_{h}+T(\tilde{N}+\tilde{M})\left(\|Q\|+L_{f}+T L_{p}\right)+$ $K_{T} \sum_{k=1}^{m}\left[\left(\tilde{M}+\tilde{N}_{1}\right) L_{1}+(\tilde{M}+\tilde{N}) \tilde{L}_{1}\right]$,

where $\gamma=T(\tilde{M}+\tilde{N}) M_{1} M_{2}$ and $\max \{\rho, \tilde{\rho}\}<1$.

Then the system (38-41) is controllable on $J$.

Proof of the above theorem is based on the application of the Banach fixed point theorem.

The authors of [22] utilise the evolution system framework to show the results for the special case of the system (38-41). Namely, they show the controllability results in two cases. In the first one, (38) takes the form:

$$
\frac{d^{2}}{d t^{2}} x(t)=A(t) x(t)+B u(t)+f\left(t, x_{t}\right)
$$

where $A(t): \mathscr{D}(A(t)) \subset X \rightarrow X$ is densely defined infinitesimal generator of the evolution system $\mathfrak{S}(t, s)$. In the second one, (38) takes the form:

$$
\frac{d^{2}}{d t^{2}} x(t)=A(t) x(t)+B u(t)+\int_{0}^{t} e(t-s) g\left(s, x_{s}\right) d s
$$

where $t \in J, t \neq t_{k}$ and $g: J \times \mathfrak{B} \rightarrow X, e: J \rightarrow X, 0 \leq s \leq t \leq T$ are appropriate functions. It is again a semilinear evolution system, but with the integrodifferential part.

4.7. Approximate controllability of impulsive systems. The approximate controllability of second order impulsive differential systems is discussed rather scarcely in the literature. In [78] the authors study the approximate controllability of impulsive semilinear evolution equations with nonlocal conditions, using the measure of noncompactness and Mönch fixed point theorem, under assumption that the nonlinear terms $f, I_{k}$ do not depend on the control variable. In [79] the authors analyse approximate controllabillity for semilinear impulsive system representing strongly damped wave equation. The analysis is performed by means of Rothe's fixed point theorem. This last work is especially interesting as it presents controllability results for mathematical model of a physical system.

Consider the following semilinear impulsive strongly damped wave equation with Dirichlet boundary conditions:

$$
\begin{cases}\frac{\partial^{2}}{\partial t^{2}} w+\eta(-\Delta)^{1 / 2} \frac{\partial}{\partial t} w+\gamma(-\Delta) w= & \\ =1_{w} u(t, x)+f\left(t, w, \frac{\partial}{\partial t} w, u(t)\right) & \text { in }(0, T) \times \Omega, \\ w=0 & \text { in }(0, T) \times \partial \Omega, \\ w(0, x)=w_{0}(x), \frac{\partial}{\partial t} w(0, x)=w_{1}(x) \text { in } \Omega, \\ \frac{\partial}{\partial t} w\left(t_{k}^{+}, x\right)=\frac{\partial}{\partial t} w\left(t_{k}^{-}, x\right)+ \\ +I_{k}\left(t_{k}, w\left(t_{k}, x\right), \frac{\partial}{\partial t} w\left(t_{k}, x\right), u\left(t_{k}, x\right)\right),\end{cases}
$$

in the space $Z_{1 / 2}=\mathscr{D}\left((-\Delta)^{1 / 2}\right) \times L^{2}(\Omega), k=1,2, \ldots, m$, where:

$1 . \Omega$ is a bounded domain in $\mathbb{R}^{n},(n \geq 1), \omega$ is an open nonempty subset of $\Omega$,

2. $1_{\omega}$ denotes the characteristic function of the set $\omega$,

3. the distributed control signal $u \in C\left([0, T], L^{2}(\Omega)\right)$,

4. $\eta, \gamma$ are positive numbers

5. $f, I_{k} \in C([0, T] \times \mathbb{R} \times \mathbb{R}, \mathbb{R}), k=1,2, \ldots, m$ are such that:

$$
\begin{aligned}
& |f(t, w, v, u)|<a_{0}\left(|w|^{\alpha_{0}}+|v|^{\alpha_{0}}\right)+b_{0}|u|^{\beta_{0}}+c_{0}, \\
& u, w, v \in \mathbb{R}
\end{aligned}
$$

$\left|I_{k}(t, w, v, u)\right|<a_{k}\left(|w|^{\alpha_{k}}+|v|^{\alpha_{k}}\right)+b_{k}|u|^{\beta_{k}}+c_{k}$,

$u, w, v \in \mathbb{R}$,

where $\frac{1}{2} \leq \alpha_{k} \leq 1, \frac{1}{2} \leq \beta_{k} \leq 1$ and

$w\left(t_{k}, x\right)=w\left(t_{k}^{+}, x\right)=\lim _{t \rightarrow t_{k}^{+}} w(t, x), w\left(t_{k}^{-}, x\right)=\lim _{t \rightarrow t_{k}^{-}} w(t, x)$,

$\frac{\partial}{\partial t} w\left(t_{k}, x\right)=\frac{\partial}{\partial t} w\left(t_{k}^{+}, x\right)=$

$=\lim _{t \rightarrow t_{k}^{+}} \frac{\partial}{\partial t} w(t, x), \frac{\partial}{\partial t} w\left(t_{k}^{-}, x\right)=\lim _{t \rightarrow t_{k}^{-}} \frac{\partial}{\partial t} w(t, x)$.

To write the abstract formulation of (45) let $X=L^{2}(\Omega, \mathbb{R})$ and consider the linear unbounded operator $A: \mathscr{D}(A) \subset X \rightarrow X$ defined by $A \phi=-\Delta \phi$, where $\mathscr{D}(A)=H^{2}(\Omega, \mathbb{R}) \cap H_{0}^{1}(\Omega, \mathbb{R})$. With the change of variables $\frac{\partial}{\partial t} w=v$, authors of [79] reformulate (45) into a first order system with the state being a member of $Z=X^{1 / 2} \times X$, namely:

$\left\{\begin{array}{l}\frac{\partial}{\partial t} z=\mathscr{A} z+B_{w} u+F(t, z, u(t)), t \in(0, T], t \neq t_{k}, \\ z(0)=z_{0}, \\ z\left(t_{k}^{+}\right)=z\left(t_{k}^{-}\right)+J_{k}\left(t_{k}, z\left(t_{k}\right), u\left(t_{k}\right)\right), k=1,2, \ldots, m\end{array}\right.$

where:

$z=\left[\begin{array}{c}w \\ v\end{array}\right], B_{w}=\left[\begin{array}{c}0 \\ 1_{w} I\end{array}\right]$ and $\mathscr{A}=\left[\begin{array}{cc}0 & I_{X} \\ -\gamma A & -\eta A^{1 / 2}\end{array}\right]$ 
is an ubounded linear operator with domain $\mathscr{D}(A)=\mathscr{D}(A) \times$ $\mathscr{D}\left(A^{1 / 2}\right)$ and $J_{k}, F:[0, T] \times Z \times U \rightarrow Z$ are defined by

$F(t, z, u)=\left[\begin{array}{c}0 \\ f(t, w, v, u)\end{array}\right]$ and $J_{k}(t, z, u)=\left[\begin{array}{c}0 \\ I_{k}(t, w, v, u)\end{array}\right]$.

According to [80] the operator $\mathscr{A}$ generates a strongly continuous semigroup $\{T(t)\}_{t>0}$ in the space $Z$. Denote the controllability map $G: L^{2}([0, T), U) \rightarrow Z$ given by $G u=\int_{0}^{T} T(T-s)$ $B_{w} u(s) d s$ whose adjoint $G^{*}: Z \rightarrow L^{2}([0, T], U)$ is given by $\left(G^{*} z\right)(s)=B_{w}^{*} T^{*}(T-s) z$ for every $s \in[0, T]$ and every $z \in Z$.

The mild solution in this case takes the form:

$$
\begin{aligned}
z_{\alpha}(t) & =T(t) z_{0}+\int_{0}^{t} T(T-s) B_{w} u_{\alpha}(s) d s \\
& +\int_{0}^{t} T(T-s) F\left(s, z_{\alpha}(s) u_{\alpha}(s)\right) d s \\
& +\sum_{0<t_{k}<t} T\left(t-t_{k}\right) J_{k}\left(t_{k}, z_{\alpha}\left(t_{k}\right), u_{\alpha}\left(t_{k}\right)\right) .
\end{aligned}
$$

In the above setting the following theorem is true.

Theorem 4.14. Approximate controllability of the abstract damped wave equation [79]. The nonlinear system [48] is approximately controllable on $[0, T]$, and a sequence of controls steering this system from the initial state $z_{0}$ to an $\varepsilon$-neighbourhood of the final stata $z_{1}$ at time $T>0$ is given by:

$$
u_{\alpha}(t)=B_{w}^{*} T^{*}(T-s)\left(\alpha I+G G^{*}\right)^{-1} \mathscr{L}\left(z_{\alpha}, u_{\alpha}\right)
$$

and the error $E_{\alpha} z$ of this approximation is given by:

$$
E_{\alpha} z=\alpha\left(\alpha I+G G^{*}\right)^{-1} \mathscr{L}\left(z_{\alpha}, u_{\alpha}\right)
$$

The proof of the above theorem is based on Rothe's fixed point theorem [81].

\section{Related problems}

It is a well known fact that there is a strong relationship between concepts of controllability and stabilizability for finite dimensional time-invariant undelayed systems that can be stated as follows: A finite dimensional time-invariant continuous-time undelayed dynamical system is stabilizable if and only if all unstable modes of this system are controllable [7, 82]. However, it has to be emphasized that for infinite dimensional systems the problem of establishing such relationships is much more difficult. It is mainly related to the fact that there are different definitions of stability for infinite dimensional systems that depend strongly on the chosen topology. There is also a great number of publications devoted to the controllability of discrete-time and discrete-continuous dynamical systems [7, 82, 83]. Control problems of finite-dimensional discrete-time systems are discussed in [84]. An overview of results related to controllability of discrete-time systems defined both, in finite-dimensional and infinite-dimensional spaces may be found in [85]. There, prob- lems for discrete-time systems are discussed, including those related to stationary and nonstationary systems, systems with multiple delays in control and state, systems with additive disturbances, descriptor systems and constrained controllability of nonlinear systems. In addition, sufficient conditions are derived for weak asymptotic stabilizability of discrete-time convex processes expressed in terms of constrained controllability conditions. For a detailed analysis of these aspects, we refer the reader to references mentioned above.

\section{Conclusions}

In this paper, a survey on the newest controllability results for various types of second order dynamical systems has been given. A variety of criteria for recognizing systems controllability have been presented. Some of them have the form of easy-to-test conditions that are significant both from practical and theoretical point of view. The exposition of results has been focused on a presentation of different approaches to the problem of controllability analysis. As this subject is already a mature discipline, its body of literature is considerable. For this reason it was not feasible to tackle every aspect of it. In particular, the paper is not focused on any specific method of controllability analysis. However, it is inevitable that some methods are more versatile than others and bring constructive results more often. Apart from the moments method, mentioned for coupled hyperbolic equations system, special attention is drawn to analysis methods based on fixed point approach. The fixed point method, mostly in combination with measures of noncompactness, finds its application in analysis of approximate and exact controllability problem for nonlinear deterministic as well as stochastic dynamical systems (see for example $[50,79,51,77,86]$ and references therein, together with references we provided throughout Section 4). Results presented within this paper have shown the complexity of controllability analysis for the systems under discussion. Therefore, it should be pointed out that there are many open problems for controllability concepts for special types of dynamical systems (especially nonlinear systems) that can be easily stated but the answer to them is unknown up to the present time.

Acknowledgements. The research presented here by Jerzy Klamka and Radoslaw Zawiski was done by authors as part of the projects funded by the National Science Centre in Poland granted according to decisions DEC-2012/07/B/ST7/01404 and DEC-2014/13/B/ST7/00755, respectively. Moreover, Janusz Wyrwal's work was supported by Polish Ministry for Science and Higher Education under internal grant BK-213/RAU1/2016 for Institute of Automatic Control, Silesian University of Technology, Gliwice, Poland.

\section{REFERENCES}

[1] R. E. Kalman, "Contributions to the theory of optimal control", Boletin de la Sociedad Matematica Mexicana 5, 102-119 (1960).

[2] R. E. Kalman, "On general theory of control systems", in: Proceedings of the 1th IFAC Congress, 481-493 (1960). 
[3] R. E. Kalman, "Canonical structure of linear dynamical systems", Proceedings of the National Academy of Sciences of the United States of America 48, 596-600 (1962).

[3] R.E. Kalman, "Canonical structure of linear dynamical systems", Proceedings of the National Academy of Sciences of the United States of America 48 (4), 596-600 (1962).

[4] R.E. Kalman, "Mathematical description of linear dynamical systems", Journal of SIAM Series A Control 1 (2), 152-192 (1963).

[5] R.E. Kalman, P.L. Falb, and M.A. Arbib, Topics in Mathematical System Theory, McGraw-Hill, New York, 1969.

[6] C.T. Chen, Introduction to Linear Systems Theory, Holt, Rinehart and Winston Inc., New York, 1970.

[7] J. Klamka, Controllability of Dynamical Systems, Kluwer Academic, Dordrecht, 1991.

[8] B. De Schutter, "Minimal state-space realization in linear system theory: an overview", Journal of Computational and Applied Mathematics 121 (1-2), 331-354 (2000).

[9] B.C. Moore, "Principal component analysis in linear systems: controllability, observability and model reduction", IEEE Transactions on Automatic Control 26 (1), 17-31 (1981).

[10] L.M. Silverman, "Realization of linear dynamical systems", IEEE Transactions on Automatic Control 16 (6), 54-567 (1971)

[11] E.G. Gilbert, "Controllability and observability in multivariable control systems", Journal of SIAM Series A Control 1 (2), 128-151 (1963).

[12] D.G. Luenberger, "Canonical forms for linear multivariable systems", IEEE Transactions on Automatic Control 12 (3), 290-290 (1967).

[13] M.K. Solak, "Transformations between canonical forms for multivariable linear constant systems", International Journal of Control 40 (1), 141-148 (1984).

[14] J. Klamka, "Minimum energy control of discrete systems with delays in control", International Journal of Control 26 (5), 737-744 (1977).

[15] J. Klamka, "Minimum energy control of 2-D systems in Hilbert spaces", Systems Science 9 (1-2), 33-42 (1983).

[16] J. Klamka, "Stochastic controllability and minimum energy control of systems with multiple delays in control", Applied Mathematics and Computation 206 (2), 704-715 (2008).

[17] T. Kaczorek and J. Klamka, "Minimum energy control of 2-D linear systems with variable coefficients", International Journal of Control 44 (3), 645-650 (1986).

[18] A. Pazy, Semigroups of Linear Operators and Applications to Partial Differential Equations, Springer-Verlag, New York, 1983.

[19] R. Triggiani, "On the lack of exact controllability for mild solutions in Banach spaces", Journal of Mathematical Analysis and Applications 50 (2), 438-446 (1975).

[20] Y. Hino, S. Murakami, and T. Naito, Functional Differential Equations with Infinite Delay, Springer, Berlin, 1991.

[21] J.K. Hale and J. Kato, "Phase space for retarded equations with infinite delay", Funkcialaj Ekvacioj 21, 11-41 (1978).

[22] G. Arthi and K. Balachandran, "Controllability of second-order impulsive evolution systems with infinite delay", Nonlinear Analysis: Hybrid Systems 11 (1), 139-153 (2014).

[23] L. Caccetta and V.G. Rumchev, "A survey of reachability and controllability for positive linear systems", Annals of Operations Research 98 (1), 101-122 (2000).

[24] C.J. Wang, "Controllability and observability of linear time varying singular systems", IEEE Transactions on Automatic Control 44 (10), 1901-1905 (1999).

[25] L. Pandolfi, "Controllability and stabilization for linear systems of algebraic and differential equations", Journal of Optimization Theory \& Applications 30 (4), 601-620 (1980).
[26] L. Armentano, "The pencil (sE-A) and controllability-observability for generalized linear systems: a geometric approach", SIAM Journal on Control Optimization 24 (4), 616-638 (1986).

[27] L. Banaszuk and K.M. Przyłuski, "On perturbations of controllable implicit linear systems", IMA Journal of Mathematical Control and Information 16 (1), 91-102 (1999).

[28] D.J. Bender and A.J. Laub, "Controllability and observability at infinity of multivariable linear second-order models", IEEE Transactions on Automatic Control 30 (12), 1234-1237 (1985).

[29] T. Berger, and T. Reis, Surveys in Differential-Algebraic Equations I, Springer-Verlag, Berlin, 2013.

[30] R.E. O'Brien, "Perturbation of controllable systems", SIAM Journal on Control and Optimization 17 (2), 462-491 (1975).

[31] T. Kobayashi, "Simplified conditions of controllability and observability for a model of flexible structures", Archives of Control Sciences 4, 251-259 (1995).

[32] J. Klamka and J.Wyrwał, "Controllability of second-order infinite-dimensional systems", Systems and Control Letters 57 (5), 386- 391 (2008).

[33] A. Bashirov and M. Jneid, "On partial complete controllability of semilinear systems", Abstract and Applied Analysis 2013, 1-8 (2013).

[34] H. Leiva, N. Merentes, and J. Sanchez, "A characterization of semilinear dense range operators and applications", Abstract and Applied Analysis 2013, 1-11 (2013).

[35] H. Leiva, N. Merentes, and J. Sanchez, "Approximate controllability of semilinear reaction diffusion equations", Mathematical Control and Related Fields 2 (2), 171-182 (2012).

[36] R. Sakthivel, R. Ganesh, Y. Ren, and S. Anthoni, "Approximate controllability of nonlinear fractional dynamical systems", Communications in Nonlinear Science and Numerical Simulation 18 (12), 3498-3508 (2013).

[37] J. Klamka, "Constrained controllability of semilinear systems", Nonlinear Analysis 47 (5), 2939-2949 (2001).

[38] J. Klamka, "Constrained controllability of semilinear systems with multiple delays in control", Bull. Pol. Ac.: Tech. 52 (1), 25-30 (2004).

[39] J. Klamka, "Constrained controllability of semilinear systems with delays", Nonlinear Dynamics 56 (1), 169-177 (2009).

[40] J. Klamka, "Constrained controllability of second order dynamical systems with delay", Proceedings of the 14th International Conference on Methods and Models in Automation and Robotics, 15-20 (2009).

[41] J.Y. Park, K. Balachandran, and G. Arthi, "Controllability of impulsive neutral integrodifferential systems with infinite delay in Banach spaces", Nonlinear Analysis: Hybrid Systems 3 (3), 184-194 (2009).

[42] J. Klamka, "Constrained controllability of semilinear systems with delayed controls", Bull. Pol. Ac.: Tech. 56 (4), 333-337 (2008).

[43] D.N. Chalishajar and H. Chalishajar, "Trajectory controllability of second order nonlinear integro-differential system: An analytical and a numerical estimation", Differential Equations and Dynamical Systems 23 (4), 467-481 (2015).

[44] N.I. Mahmudov and S.J. Zorlu, "Controllability of nonlinear stochastic systems", International Journal of Control 76 (2), 95-104 (2003).

[45] N.I. Mahmudov and A. Denker, "On controllability of linear stochastic systems", International Journal of Control 73 (2), 144-151 (2000).

[46] J. Klamka, "Stochastic controllability of linear systems with delay in control", Bull. Pol. Ac.: Tech. 55 (1), 23-29 (2007). 
[47] J. Klamka, "Stochastic controllability of systems with variable delay in control", Bull. Pol. Ac.: Tech. 56 (3), 279-284 (2008).

[48] J. Klamka, "Stochastic controllability of linear systems with state delays", International Journal of Applied Mathematics and Computer Science 17 (1), 5-13 (2007).

[49] J. Klamka, "Controllability of dynamical systems. A survey", Bull. Pol. Ac.: Tech. 61 (2), 335-342 (2013).

[50] S. Das, D. Pandey, and N. Sukavanam, "Existence of solution and approximate controllability of a second-order neutral stochastic differential equation with state dependent delay", Acta Mathematica Scientia 36 (5), 1509-1523 (2016).

[51] U. Arora and N. Sukavanam, "Approximate controllability of second order semilinear stochastic system with variable delay in control and with nonlocal conditions", Rendiconti del Circolo Matematico di Palermo 65 (2), 307-322 (2016).

[52] P. Muthukumar and C. Rajivganthi, "Approximate controllability of second-order neutral stochastic differential equations with infinite delay and poisson jumps", Journal of Systems Science \& Complexity 28 (5), 1033-1048 (2015).

[53] G. Chen and X. Dong, "On feedback control of chaotic continuous time systems", IEEE Transactions on Circuits Systems I: Fundamental Theory and Applicatons 40 (9), 591-601 (1993).

[54] H. Nijmeijer and H. Berghuis, "On Lyapunov control of the duffing equation", IEEE Transactions on Circuits Systems I: Fundamental Theory and Applicatons 42 (8), 473-477 (1995).

[55] G. Duffing, Erzwungene Schwingungen bei Veränderlicher Eigenfrequenz, F. Vieweg \& Sohn, Braunschweig, 1918.

[56] N. Van Loi and V. Obukhovskii, "On controllability of duffing equation", Applied Mathematics and Computation 219 (29), 10468- 10474 (2013).

[57] A. Ammar-Kohdia, M. Benabdallah, L. Gonzáles-Burgos, and L. de Teresa, "Recent results on the controllability of coupled parabolic problems: a survey", Mathematical Control and Related Fields 1 (3), 267-306 (2011).

[58] R. Dáger, "Insensitizing controls for the 1-d wave equation", SIAM J. Control Optim. 45 (5), 1758-1768 (2006).

[59] L. Tebou, "Locally distributed desensitizing controls for the wave equation", C. R. Math. Acad. Sci. Paris 346 (7), 407-412 (2008).

[60] F. Alabau-Boussouira, "A two-level energy method for indirect boundary observability and controllability of weakly coupled hyperbolic systems", SIAM J. Control Optim. 42 (3), 871-906 (2003).

[61] F. Alabau-Boussouira and M. Leautaud, "Indirect controllability of locally coupled systems under geometric conditions", $C$. R. Math. Acad. Sci. Paris 349 (7), 395-400 (2011).

[62] D. Russell, "Controllability and stabilizability theory for linear partial differential equations", SIAM Review 20 (4), 639-739 (1978).

[63] S. Avdonin and S. Ivanov, Families of Exponentials. The Method of Moments in Controllability Problems for Distributed Parameter Systems, Cambridge University Press, New York, 1995.

[64] S. Hansen and E. Zuazua, "Exact controllability and stabilization of a vibrating spring with an interior point mass", SIAM J. Control Optim. 33 (5), 1357-1391 (1995).

[65] S. Avdonin, A. Choque Rivero, and L. de Teresa, "Exact boundary controllability of coupled hyperbolic equations", International Journal of Applied Mathematics and Computer Science 23 (4), 701-710 (2013).
[66] A. Gallo and A. Piccirillo, "About new analogies of Gronwall-Bellman- Bihari type inequalities for discontinuous functions and estimated solutions for impulsive differential systems", Nonlinear Analysis 67 (5), 1550-1559 (2007).

[67] V. Lakshmikantham, D.D. Bainov, and P.S. Simeonov, Theory of Impulsive Differential Equations, World Scientific, Singapore, 1989.

[68] Y. Rogovchenko, "Impulsive evolution systems: main results and new trends", Dynamics of Continuous, Discrete and Impulsive Systems 3 (1), 57-88 (1997).

[69] A. Samoilenko and N. Perestyuk, Impulsive Differential Equations, World Scientific, Singapore, 1995.

[70] S. Zavalishchin and A. Sesekin, Dynamic Impulse Systems. Theory and Applications, Kluwer Academic Publishers Group, Dordrecht, 1997.

[71] M. Choisy, J.F. Guegan, and P. Rohani, "Dynamics of infectious diseases and pulse vaccination: tearing apart the embedded resonance effect", Physica D 223 (1), 26-35 (2006).

[72] W. Wang, H. Wang, and Z. Li, "The dynamic complexity of a three-species Beddington-type food chain with impulsive control strategy", Chaos Solitions and Fractals 32 (5), 1772-1785 (2007).

[73] W. Zhang and M. Fan, "Periodicity in a generalized ecological competition system governed by impulsive differential equations with delays", Mathematical and Computer Modelling 39 (4-5), 479- 493 (2004).

[74] J.K. Hale and J. Kato, "Phase space for retarded equations with infinite delay", Funkcialaj Ekvacioj 21, 11-41 (1978).

[75] F. Kappel and W. Schappacher, "Some considerations to the fundamental theory of infinite delay equations", Journal of Differential Equations 37 (2), 141-183 (1980).

[76] J.K. Hale and S.M.V. Lunel, Introduction to Functional Differential Equations, Springer-Verlag, New York, 1993.

[77] G. Arthi and J. Park, "On controllability of second-order impulsive neutral integrodifferential systems with infinite delay", IMA Journal of Mathematical Control and Information 32 (3), 639-657 (2015).

[78] L. Chen and G. Li, "Approximate controllability of impulsive differential equations with nonlocal conditions", International Journal of Nonlinear Science 10 (4), 438-446 (2010).

[79] H. Larez, H. Leiva, J. Rebaza, and A. Rios, "Approximate controllability of semilinear impulsive strongly damped wave equation", Journal of Applied Analysis 21 (1), 45-58 (2015).

[80] S. Chen and R. Triggiani, "Proof of extensions of two conjectures on structural damping for elastic systems", Pacific Journal of Mathematics 136 (1), 15-55 (1989).

[81] J. Bana's and K. Goebel, Measures of Noncompactness in Banach Spaces, Marcel Dekker, New York, 1980.

[82] W. Mitkowski, Stabilization of Dynamical Systems, WNT, Warsaw, 1991.

[83] W. Mitkowski, W. Bauer, and M. Zagórowska, "Discrete-time feedback stabilization", Proceedings of the 20th National Conference on Automation of Discrete Processes, (2016).

[84] J.P. LaSale, The Stability and Control of Discrete Processes, Springer-Verlag, New York, 1986.

[85] V.N. Phat, Constrained Control Problems of Discrete Processes, World Scientific, Singapore, 1996.

[86] R. Zawiski, "On controllability and measures of noncompactness", Proceedings of the 10th International Conference on Mathematical Problems in Engineering, Aerospace and Sciences: ICNPAA 2014 1637, 1241- 1246 (2014). 